\title{
Development of a Library of 6-Arylcoumarins as Candidate Fluorescent Sensors
}

\author{
Tomoya Hirano*, Kenichi Hiromoto, Hiroyuki Kagechika* \\ School of Biomedical Science, Institute of Biomaterials and Bioengineering, Tokyo Medical and \\ Dental University, 2-3-10 Kanda-Surugadai, Chiyoda-ku, Tokyo 101-0062, Japan. \\ E-mail: hiraomc@tmd.ac.jp, kageomc@tmd.ac.jp
}

\section{Supporting Information}

\section{Materials and Reagents}

All reagents were purchased from Sigma-Aldrich Chemical Co., Tokyo Kasei Kogyo Co, Wako Pure Chemical Industries, and Kanto Kagaku Co., Inc. Silica gel for column chromatography was purchased from Kanto Kagaku Co., Inc.. Extracted solutions were dried over anhydrous $\mathrm{Na}_{2} \mathrm{SO}_{4}$ and solvents were evaporated under reduced pressure. ${ }^{1} \mathrm{H}$ and ${ }^{13} \mathrm{C}$ NMR spectra were recorded on a Bruker Advance 500 spectrometer. Mass spectral data was obtained on JEOL JMS-T1000LC AccuTOF or Brucker Daltonics microTOF-2focus in the positive and negative ion detection modes. Melting points were taken on a Yanagimoto micro melting point apparatus and are uncorrected. Elemental analyses were carried out by Yanaco MT-6 CHN CORDER spectrometer. UV spectra were recorded with JASCO V-550, and fluorescence spectra were recorded with JASCO FP-6600.

Ethyl 6-bromo-7-(diethylamino)-2-oxo-2H-chromene-3-carboxylate 1.

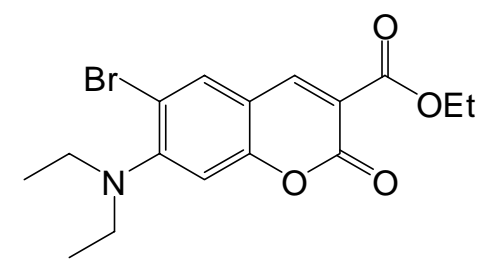

1

Compound 1 was prepared from 4-(diethylamino)salicylaldehyde according to the procedure described for the reference ${ }^{13}$.

$\mathrm{Mp} 76-77^{\circ} \mathrm{C}$ (lit. $\left.74.5^{-76}{ }^{\circ} \mathrm{C}\right) ;{ }^{1} \mathrm{H}$ NMR $\left(500 \mathrm{MHz} \mathrm{CDCl}_{3}\right) \delta 8.36(\mathrm{~s}, 1 \mathrm{H}), 7.72(\mathrm{~s}, 1 \mathrm{H}), 6.85(\mathrm{~s}, 1 \mathrm{H})$, 4.36 (q, $J=7.1 \mathrm{~Hz}, 2 \mathrm{H}), 3.28$ (q, $J=7.0 \mathrm{~Hz}, 4 \mathrm{H}), 1.36(\mathrm{t}, J=7.1 \mathrm{~Hz}, 3 \mathrm{H}), 1.11(\mathrm{t}, J=7.0 \mathrm{~Hz}, 6 \mathrm{H})$; ${ }^{13} \mathrm{C} \mathrm{NMR}\left(125 \mathrm{MHz}, \mathrm{CDCl}_{3}\right) \delta 163.1,156.6,155.4,155.0,147.3,134.3,114.6,113.5,112.8,108.6$, 61.6, 45.9 (2C), 14.1, 12.2 (2C); HRMS (ESI) calcd for $\mathrm{C}_{16} \mathrm{H}_{18} \mathrm{BrNO}_{4} \mathrm{Na}(\mathrm{M}+\mathrm{Na})+390.0317$. Found 390.0307 . 
General Procedure: Pd-Catalyzed Suzuki-Miyaura Coupling.

Ethyl 7-(diethylamino)-2-oxo-6-phenyl-2H-chromene-3-carboxylate 3a.

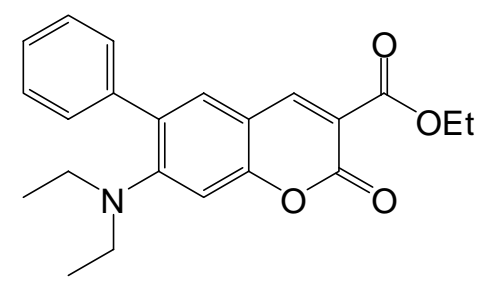

$3 a$

To a stirred solution of $2 \mathrm{a}(34.3 \mathrm{mg}, 0.168 \mathrm{mmol}), \mathrm{CsF}(43.5 \mathrm{mg}, 0.286 \mathrm{mmol})$ and $\mathrm{PdCl}_{2}(\mathrm{dppf}) \cdot \mathrm{CH}_{2} \mathrm{Cl}_{2}(9.4 \mathrm{mg}, 0.012 \mathrm{mmol})$ in dry DMF $(1 \mathrm{ml})$ was added 1 (20.3 mg, $\left.0.055 \mathrm{mmol}\right)$ at room temperature under an atmosphere of argon. The reaction mixture was heated under $60^{\circ} \mathrm{C}$ for $2 \mathrm{~h}$, cooled to room temperature. The resultant mixture was diluted with EtOAc, washed with saturated aqueous $\mathrm{NH}_{4} \mathrm{Cl}$, water and brine, dried, and concentrated. Purification of the residue by flash column chromatography (silica gel, 20\% EtOAc/hexane) gave 3a (14.5 mg, 72\%) as yellow cubes. A small amount of this compound was recrystallized in $i-\operatorname{Pr}_{2} \mathrm{O}$ to give an analytical sample.

$\mathrm{Mp} 123-124^{\circ} \mathrm{C} ;{ }^{1} \mathrm{H}$ NMR $\left(500 \mathrm{MHz} \mathrm{CDCl}_{3}\right) \delta 8.44(\mathrm{~s}, 1 \mathrm{H}), 7.40(\mathrm{~m}, 4 \mathrm{H}), 7.31(\mathrm{~m}, 1 \mathrm{H}), 7.28(\mathrm{~s}, 1 \mathrm{H})$, $6.85(\mathrm{~s}, 1 \mathrm{H}), 4.37(\mathrm{q}, J=7.1 \mathrm{~Hz}, 2 \mathrm{H}), 3.03(\mathrm{q}, J=7.1 \mathrm{~Hz}, 4 \mathrm{H}), 1.37(\mathrm{t}, J=7.1 \mathrm{~Hz}, 3 \mathrm{H}), 0.95(\mathrm{t}, J=$ $7.0 \mathrm{~Hz}, 6 \mathrm{H}) ;{ }^{13} \mathrm{C} \mathrm{NMR}\left(125 \mathrm{MHz}, \mathrm{CDCl}_{3}\right) \delta 163.8,157.6,156.4,155.2,148.8,140.5,132.7,131.6$, 128.7 (2C), 128.2 (2C), 127.3, 112.8, 111.1, 105.7, 61.4, 45.8 (2C), 14.3, 12.1 (2C); HRMS (ESI) calcd for $\mathrm{C}_{22} \mathrm{H}_{23} \mathrm{NO}_{4} \mathrm{Na}(\mathrm{M}+\mathrm{Na})+388.1525$. Found 388.1502; Anal. Calcd for $\mathrm{C}_{22} \mathrm{H}_{23} \mathrm{NO}_{4}$ : C, 72.31 ; H, 6.34; N, 3.83. Found: C, 72.33; H, 6.45; N, 3.56.

Ethyl 6-(4-aminophenyl)-7-(diethylamino)-2-oxo-2H-chromene-3-carboxylate $3 \mathrm{~b}$.

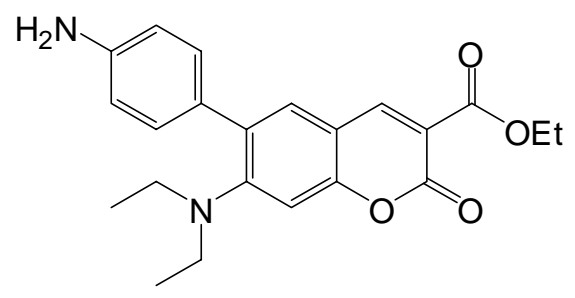

$3 b$

Compound $3 \mathrm{~b}$ was prepared from $2 \mathrm{~b}$ according to the procedure described for $3 \mathrm{a}$. Yield $85 \%$ (a pale orange powder). A small amount of this compound was recrystallized in $\mathrm{MeOH} / i-\mathrm{Pr}_{2} \mathrm{O}$ to give an analytical sample.

Mp 170-171 ${ }^{\circ} \mathrm{C} ; 1 \mathrm{H} \mathrm{NMR}\left(500 \mathrm{MHz} \mathrm{CDCl}_{3}\right) \delta 8.42(\mathrm{~s}, 1 \mathrm{H}), 7.23(\mathrm{~s}, 1 \mathrm{H}), 7.21(\mathrm{~d}, J=8.4 \mathrm{~Hz}, 2 \mathrm{H})$, $6.80(\mathrm{~s}, 1 \mathrm{H}), 6.73(\mathrm{~d}, J=8.3 \mathrm{~Hz}, 2 \mathrm{H}), 4.36$ (q, $J=7.1 \mathrm{~Hz}, 2 \mathrm{H}), 3.76(\mathrm{br} \mathrm{s}, 2 \mathrm{H}), 3.05$ (q, $J=7.1 \mathrm{~Hz}$, 
$4 \mathrm{H}), 1.36(\mathrm{t}, J=7.1 \mathrm{~Hz}, 3 \mathrm{H}), 0.95(\mathrm{t}, J=7.1 \mathrm{~Hz}, 6 \mathrm{H}) ;{ }^{13} \mathrm{C} \mathrm{NMR}\left(125 \mathrm{MHz}, \mathrm{CDCl}_{3}\right) \delta 163.8,157.8$, 156.1, 155.4, 148.9, 145.2, 132.3, 131.7, 130.8, 129.1 (2C), 115.5 (2C), 112.4, 111.1, 105.6, 61.4, $45.5(2 \mathrm{C}), 14.3,12.1$ (2C); HRMS (ESI) calcd for $\mathrm{C}_{22} \mathrm{H}_{24} \mathrm{~N}_{2} \mathrm{O}_{4} \mathrm{Na}(\mathrm{M}+\mathrm{Na})^{+}$403.1634. Found 403.1608; Anal. Calcd for $\mathrm{C}_{22} \mathrm{H}_{24} \mathrm{~N}_{2} \mathrm{O}_{4} \cdot 1 / 8 \mathrm{H}_{2} \mathrm{O}$ : C, 69.05; H, 6.39; N, 7.32. Found: C, 69.05; H, 6.36; N, 7.34 .

Ethyl 6-(4-acetamidophenyl)-7-(diethylamino)-2-oxo-2H-chromene-3-carboxylate 3c.

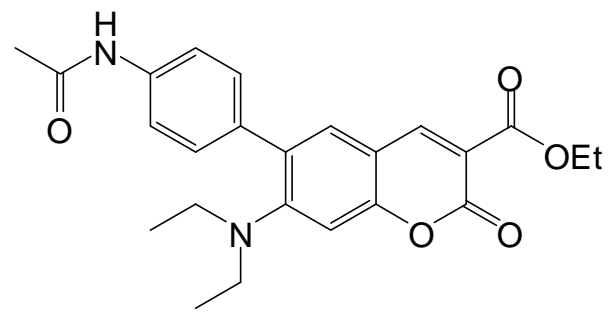

$3 \mathrm{c}$

Compound 3c was prepared from 2c according to the procedure described for $3 \mathrm{a}$. Yield $63 \%$ (yellow needles). A small amount of this compound was recrystallized in EtOAc to give an analytical sample.

$\mathrm{Mp} 125-126^{\circ} \mathrm{C} ;{ }^{1} \mathrm{H} \mathrm{NMR}\left(500 \mathrm{MHz}, \mathrm{CDCl}_{3}\right) \delta 8.43(\mathrm{~s}, 1 \mathrm{H}), 7.57(\mathrm{~d}, J=8.1 \mathrm{~Hz}, 2 \mathrm{H}), 7.48(\mathrm{~s}, 1 \mathrm{H})$, $7.38(\mathrm{~d}, J=8.2 \mathrm{~Hz}, 2 \mathrm{H}), 7.25(\mathrm{~s}, 1 \mathrm{H}), 6.82(\mathrm{~s}, 1 \mathrm{H}), 4.36(\mathrm{q}, J=7.1 \mathrm{~Hz}, 2 \mathrm{H}), 3.03(\mathrm{q}, J=7.0 \mathrm{~Hz}, 4 \mathrm{H})$, $2.19(\mathrm{~s}, 3 \mathrm{H}), 1.36(\mathrm{t}, J=7.1 \mathrm{~Hz}, 3 \mathrm{H}), 0.95(\mathrm{t}, J=7.0 \mathrm{~Hz}, 6 \mathrm{H}) ;{ }^{13} \mathrm{C} \mathrm{NMR}\left(125 \mathrm{MHz}, \mathrm{CDCl}_{3}\right) \delta 168.4$, 163.7, 157.7, 156.3, 155.3, 148.8, 137.3, 136.1, 132.6, 131.1, 128.7 (2C), 120.0 (2C), 112.8, 111.2, 105.9, 61.5, 45.7 (2C), 24.6, 14.3, 12.1 (2C); HRMS (ESI) calcd for $\mathrm{C}_{24} \mathrm{H}_{26} \mathrm{~N}_{2} \mathrm{O}_{5} \mathrm{Na}(\mathrm{M}+\mathrm{Na})^{+}$ 445.1739. Found 445.1713; Anal. Calcd for $\mathrm{C}_{24} \mathrm{H}_{26} \mathrm{~N}_{2} \mathrm{O}_{5}$ : C, 68.23; H, 6.20; N, 6.63. Found: C, 68.03; H, 6.23; N, 6.57.

Ethyl 7-(diethylamino)-6-(4-(dimethylamino)phenyl)-2-oxo-2 $H$-chromene-3-carboxylate 3d.

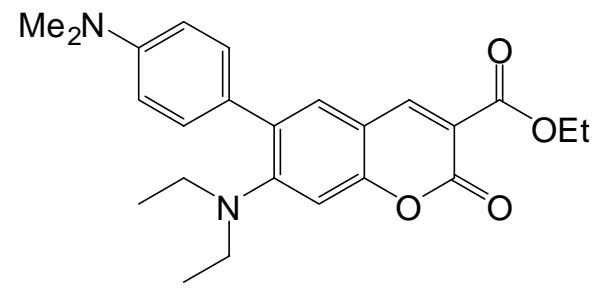

$3 d$

Compound 3d was prepared from $2 \mathrm{~d}$ according to the procedure described for $3 \mathrm{a}$. Yield $66 \%$ (yellow cubes). A small amount of this compound was recrystallized in $i-\operatorname{Pr}_{2} \mathrm{O}$ to give an analytical sample.

$\mathrm{Mp} 166-168^{\circ} \mathrm{C} ;{ }^{1} \mathrm{H} \mathrm{NMR}\left(500 \mathrm{MHz}, \mathrm{CDCl}_{3}\right) \delta 8.42(\mathrm{~s}, 1 \mathrm{H}), 7.29(\mathrm{~d}, J=8.7 \mathrm{~Hz}, 2 \mathrm{H}), 7.24(\mathrm{~s}, 1 \mathrm{H})$, 
$6.80(\mathrm{~s}, 1 \mathrm{H}), 6.73(\mathrm{~d}, J=8.4 \mathrm{~Hz}, 2 \mathrm{H}), 4.36(\mathrm{q}, J=7.1 \mathrm{~Hz}, 2 \mathrm{H}), 3.06(\mathrm{q}, J=7.1 \mathrm{~Hz}, 4 \mathrm{H}), 2.97(\mathrm{~s}, 6 \mathrm{H})$, $1.36(\mathrm{t}, J=7.1 \mathrm{~Hz}, 3 \mathrm{H}), 0.96(\mathrm{t}, J=7.1 \mathrm{~Hz}, 6 \mathrm{H}) ;{ }^{33} \mathrm{C} \mathrm{NMR}\left(125 \mathrm{MHz}, \mathrm{CDCl}_{3}\right) \delta 163.9,157.8,155.9$, 155.5, 149.6, 148.9, 132.2, 131.9, 128.7 (2C), 128.2, 112.5 (2C), 112.3, 111.1, 105.6, 61.3, 45.5 (2C), 40.4 (2C), 14.3, 12.1 (2C); HRMS (ESI) calcd for $\mathrm{C}_{24} \mathrm{H}_{28} \mathrm{~N}_{2} \mathrm{O}_{4} \mathrm{Na}(\mathrm{M}+\mathrm{Na})^{+}$431.1947. Found 431.1930; Anal. Calcd for $\mathrm{C}_{24} \mathrm{H}_{28} \mathrm{~N}_{2} \mathrm{O}_{4}$ : C, 70.57; H, 6.91; N, 6.86. Found: C, 70.41; H, 6.86; N, 6.86 .

Ethyl 7-(diethylamino)-6-(4-nitrophenyl)-2-oxo-2H-chromene-3-carboxylate 3e.

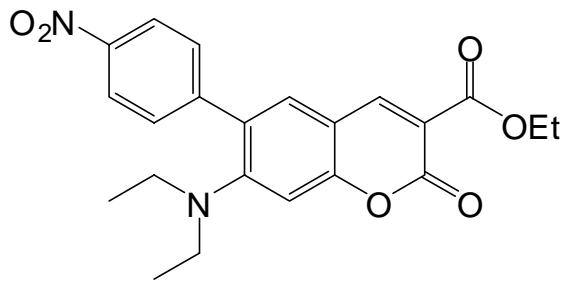

$3 e$

Compound 3e was prepared from 2e according to the procedure described for 3a. Yield 28\% (a pale yellow powder). A small amount of this compound was recrystallized in $i$ - $\operatorname{Pr}_{2} \mathrm{O}$ to give an analytical sample.

$\operatorname{Mp} 123-124^{\circ} \mathrm{C} ;{ }^{1} \mathrm{H}$ NMR $\left(500 \mathrm{MHz} \mathrm{CDCl}_{3}\right) \delta 8.45(\mathrm{~s}, 1 \mathrm{H}), 8.28(\mathrm{~d}, J=8.8 \mathrm{~Hz}, 2 \mathrm{H}), 7.64(\mathrm{~d}, J=8.8$ $\mathrm{Hz}, 2 \mathrm{H}), 7.33(\mathrm{~s}, 1 \mathrm{H}), 6.91(\mathrm{~s}, 1 \mathrm{H}), 4.38$ (q, $J=7.1 \mathrm{~Hz}, 2 \mathrm{H}), 3.03(\mathrm{q}, J=7.1 \mathrm{~Hz}, 4 \mathrm{H}), 1.38$ (t, $J=7.2$ $\mathrm{Hz}, 3 \mathrm{H}), 0.98$ (t, $J=7.1 \mathrm{~Hz}, 6 \mathrm{H}) ;{ }^{13} \mathrm{C} \mathrm{NMR}\left(125 \mathrm{MHz}, \mathrm{CDCl}_{3}\right) \delta 163.5,157.1,156.9,154.9,148.4$, 147.1, 147.0, 132.6, 129.4, 129.0 (2C), 124.2 (2C), 114.0, 111.6, 106.7, 61.7, 46.1 (2C), 14.3, 11.9 (2C); HRMS (ESI) calcd for $\mathrm{C}_{22} \mathrm{H}_{22} \mathrm{~N}_{2} \mathrm{O}_{6} \mathrm{Na}(\mathrm{M}+\mathrm{Na})^{+}$433.1376. Found 433.1381; Anal. Calcd for $\mathrm{C}_{22} \mathrm{H}_{22} \mathrm{~N}_{2} \mathrm{O}_{6} \cdot 1 / 4 \mathrm{H}_{2} \mathrm{O}: \mathrm{C}, 63.68 ; \mathrm{H}, 5.47 ; \mathrm{N}, 6.75$. Found: C, 63.90; H, 5.45; N, 6.49.

Ethyl 7-(diethylamino)-6-(4-hydroxyphenyl)-2-oxo-2H-chromene-3-carboxylate 3f.

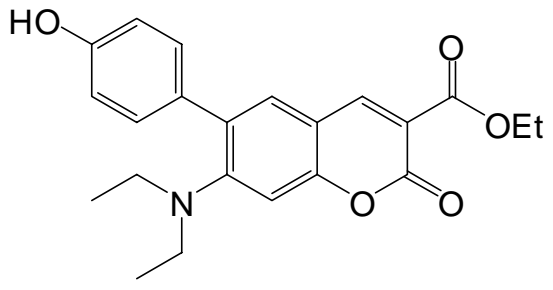

$3 f$

Compound $3 \mathrm{f}$ was prepared from $2 \mathrm{f}$ according to the procedure described for $3 \mathrm{a}$. Yield $74 \%$ (yellow needles). A small amount of this compound was recrystallized in EtOAc to give an analytical sample.

Mp $112-113^{\circ} \mathrm{C} ;{ }^{1} \mathrm{H}$ NMR $\left(500 \mathrm{MHz}, \mathrm{CDCl}_{3}\right) \delta 8.44(\mathrm{~s}, 1 \mathrm{H}), 7.29(\mathrm{~d}, J=8.6 \mathrm{~Hz}, 2 \mathrm{H}), 7.25(\mathrm{~s}, 1 \mathrm{H})$, $6.88(\mathrm{~d}, J=8.6 \mathrm{~Hz}, 2 \mathrm{H}), 6.84(\mathrm{~s}, 1 \mathrm{H}), 5,15(\mathrm{br} \mathrm{s}, 1 \mathrm{H}), 4.37(\mathrm{q}, J=7.1 \mathrm{~Hz}, 2 \mathrm{H}), 3.04(\mathrm{q}, J=7.1 \mathrm{~Hz}$, 
$4 \mathrm{H}), 1.37(\mathrm{t}, J=7.1 \mathrm{~Hz}, 3 \mathrm{H}), 0.96(\mathrm{t}, J=7.1 \mathrm{~Hz}, 6 \mathrm{H}) ;{ }^{13} \mathrm{C} \mathrm{NMR}\left(125 \mathrm{MHz}, \mathrm{CDCl}_{3}\right) \delta 163.9,158.1$, 156.2, ,155.5, 155.4, 149.1, 132.6, 132.5, 131.5, 129.4 (2C), 115.8 (2C), 112.3, 111.1, 105.6, 61.5, 45.6 (2C), 14.3, 12.1 (2C); HRMS (ESI) calcd for $\mathrm{C}_{22} \mathrm{H}_{22} \mathrm{NO}_{5}(\mathrm{M}-\mathrm{H})^{-}$380.1498. Found 380.1491; Anal. Calcd for $\mathrm{C}_{22} \mathrm{H}_{23} \mathrm{NO}_{5}$ : C, 69.28; H, 6.08; N, 3.67. Found: C, 69.02; H, 6.20; N, 3.65.

Ethyl 7-(diethylamino)-6-(4-methoxyphenyl)-2-oxo-2H-chromene-3-carboxylate 3g.

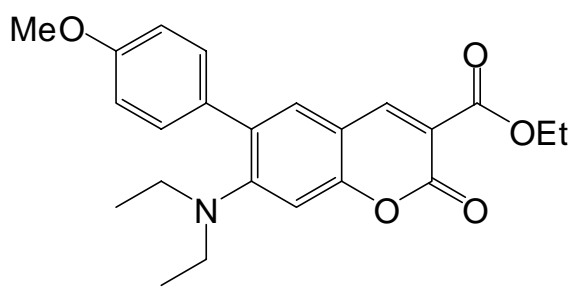

$3 \mathrm{~g}$

Compound $3 \mathrm{~g}$ was prepared from $2 \mathrm{~g}$ according to the procedure described for 3a. Yield 63\% (a pale yellow needles). A small amount of this compound was recrystallized in $i-\operatorname{Pr}_{2} \mathrm{O}$ to give an analytical sample.

Mp 70-71 ${ }^{\circ} \mathrm{C} ;{ }^{1} \mathrm{H} \mathrm{NMR}\left(500 \mathrm{MHz} \mathrm{CDCl}_{3}\right) \delta 8.43(\mathrm{~s}, 1 \mathrm{H}), 7.35(\mathrm{~d}, J=8.8 \mathrm{~Hz}, 2 \mathrm{H}), 7.26(\mathrm{~s}, 1 \mathrm{H}), 6.93$ (d, $J=8.8 \mathrm{~Hz}, 2 \mathrm{H}), 6.85(\mathrm{~s}, 1 \mathrm{H}), 4.37(\mathrm{q}, J=7.1 \mathrm{~Hz}, 2 \mathrm{H}), 3.84(\mathrm{~s}, 3 \mathrm{H}), 3.04(\mathrm{q}, J=7.1 \mathrm{~Hz}, 4 \mathrm{H}), 1.37$ (t, $J=7.1 \mathrm{~Hz}, 3 \mathrm{H}), 0.96(\mathrm{t}, J=7.1 \mathrm{~Hz}, 6 \mathrm{H}) ;{ }^{13} \mathrm{C} \mathrm{NMR}\left(125 \mathrm{MHz}, \mathrm{CDCl}_{3}\right) \delta 163.8,159.0,157.6$, 156.2, 155.1, 148.8, 132.7, 132.5, 131.4, 129.3 (2C), 114.2 (2C), 112.9, 111.3, 106.0, 61.5, 55.3, 45.8 (2C), 14.3, 12.1 (2C); HRMS (ESI) calcd for $\mathrm{C}_{23} \mathrm{H}_{25} \mathrm{NO} \mathrm{N}_{5} \mathrm{Na}(\mathrm{M}+\mathrm{Na})^{+} 418.1630$. Found 418.1608rt; Anal. Calcd for $\mathrm{C}_{23} \mathrm{H}_{25} \mathrm{NO}_{5}$ : C, 69.86; H, 6.37; N, 3.54. Found: C, 69.62; H, 6.40; N, 3.54.

Ethyl 6-(4-acetylphenyl)-7-(diethylamino)-2-oxo-2H-chromene-3-carboxylate $3 \mathrm{~h}$.

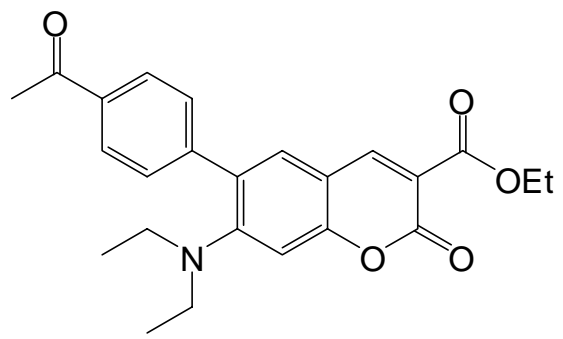

$3 h$

Compound $3 \mathrm{~h}$ was prepared from $2 \mathrm{~h}$ according to the procedure described for $3 \mathrm{a}$. Yield 58\% (a pale yellow cubes). A small amount of this compound was recrystallized in $i-\operatorname{Pr}_{2} \mathrm{O}$ to give an analytical sample.

$\mathrm{Mp} 79-80^{\circ} \mathrm{C} ;{ }^{1} \mathrm{H} \mathrm{NMR}\left(500 \mathrm{MHz}, \mathrm{CDCl}_{3}\right) \delta 8.44(\mathrm{~s}, 1 \mathrm{H}), 8.00(\mathrm{~d}, J=8.4 \mathrm{~Hz}, 2 \mathrm{H}), 7.55(\mathrm{~d}, J=8.4 \mathrm{~Hz}$, 2H), $7.30(\mathrm{~s}, 1 \mathrm{H}), 6.87(\mathrm{~s}, 1 \mathrm{H}), 4.37(\mathrm{q}, J=7.1 \mathrm{~Hz}, 2 \mathrm{H}), 3.02(\mathrm{q}, J=7.1 \mathrm{~Hz}, 4 \mathrm{H}), 2.62(\mathrm{~s}, 3 \mathrm{H}), 1.37$ (t, $J=7.1 \mathrm{~Hz}, 3 \mathrm{H}), 0.96(\mathrm{t}, J=7.1 \mathrm{~Hz}, 6 \mathrm{H}) ;{ }^{13} \mathrm{C} \mathrm{NMR}\left(125 \mathrm{MHz} \mathrm{CDCl}_{3}\right) \delta 197.4,163.6,157.3,156.7$, 
155.0, 148.6, 145.4, 136.0, 132.6, 130.4, 128.9 (2C), 128.4 (2C), 113.4, 111.3, 106.2, 61.6, 45.9 (2C), 26.6, 14.3, 12.0 (2C); HRMS (ESI) calcd for $\mathrm{C}_{24} \mathrm{H}_{25} \mathrm{NO}_{5} \mathrm{Na}(\mathrm{M}+\mathrm{Na})^{+} 430.1630$. Found 430.1606; Anal. Calcd for $\mathrm{C}_{24} \mathrm{H}_{25} \mathrm{NO}_{5}$ : C, 70.74; H, 6.18; N, 3.44. Found: C, 70.55; H, 6.29; N, 3.42.

Ethyl 6-(4-cyanophenyl)-7-(diethylamino)-2-oxo-2H-chromene-3-carboxylate 3 i.

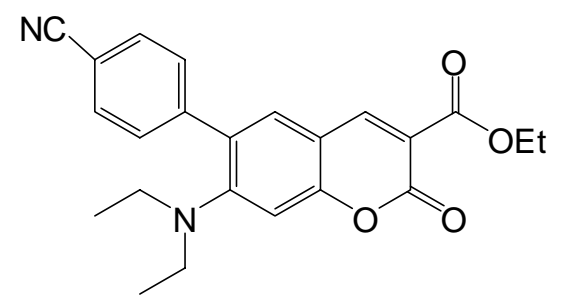

$3 \mathbf{i}$

Compound $3 \mathrm{i}$ was prepared from $2 \mathrm{i}$ according to the procedure described for $3 \mathrm{a}$. Yield $45 \%$ (pale yellow needles). A small amount of this compound was recrystallized in EtOAc to give an analytical sample.

Mp $174-176^{\circ} \mathrm{C} ;{ }^{1} \mathrm{H}$ NMR (500 $\left.\mathrm{MHz} \mathrm{CDCl}_{3}\right) \delta 8.44(\mathrm{~s}, 1 \mathrm{H}), 7.70(\mathrm{~d}, J=8.3 \mathrm{~Hz}, 2 \mathrm{H}), 7.58(\mathrm{~d}, J=8.3$ $\mathrm{Hz}, 2 \mathrm{H}), 7.29$ (s, 1H), 6.89 (s, 1H), 4.37 (q, $J=7.1 \mathrm{~Hz}, 2 \mathrm{H}), 3.01$ (q, $J=7.1 \mathrm{~Hz}, 4 \mathrm{H}), 1.37$ (t, $J=7.1$ $\mathrm{Hz}, 3 \mathrm{H}), 0.97$ (t, $J=7.0 \mathrm{~Hz}, 6 \mathrm{H}) ;{ }^{13} \mathrm{C} \mathrm{NMR}\left(125 \mathrm{MHz} \mathrm{CDCl}_{3}\right) \delta 163.5,157.2,156.8,154.9,148.5$, 145.2, 132.6 (2C), 132.6, 129.7, 128.9 (2C), 118.6, 113.7, 111.5, 111.2, 106.5, 61.6, 45.9 (2C), 14.3, 11.9 (2C); HRMS (ESI) calcd for $\mathrm{C}_{23} \mathrm{H}_{22} \mathrm{~N}_{2} \mathrm{O}_{4} \mathrm{Na}(\mathrm{M}+\mathrm{Na})+4$ 413.1477. Found 413.1462; Anal. Calcd for $\mathrm{C}_{23} \mathrm{H}_{22} \mathrm{~N}_{2} \mathrm{O}_{4}$ : C, 70.75; H, 5.68; N, 7.17. Found: C, 70.61; H, 5.75; N, 6.99.

Ethyl 7-(diethylamino)-6-(4-(methoxycarbonyl)phenyl)-2-oxo-2 $H$-chromene-3- carboxylate 3j.

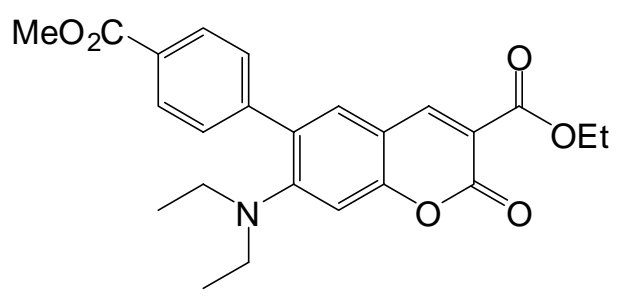

$3 \mathbf{j}$

Compound $3 \mathrm{j}$ was prepared from $2 \mathrm{j}$ according to the procedure described for $3 \mathrm{a}$. Yield $46 \%$ (yellow cubes). A small amount of this compound was recrystallized in $i-\operatorname{Pr}_{2} \mathrm{O}$ to give an analytical sample.

Mp $115-117^{\circ} \mathrm{C} ;{ }^{1} \mathrm{H}$ NMR $\left(500 \mathrm{MHz} \mathrm{CDCl}_{3}\right) \delta 8.44(\mathrm{~s}, 1 \mathrm{H}), 8.07(\mathrm{~d}, J=8.2 \mathrm{~Hz}, 2 \mathrm{H}), 7.52(\mathrm{~d}, J=8.3$ $\mathrm{Hz}, 2 \mathrm{H}), 7.31$ (s, 1H), $6.88(\mathrm{~s}, 1 \mathrm{H}), 4.37$ (q, $J=7.1 \mathrm{~Hz}, 2 \mathrm{H}), 3.93(\mathrm{~s}, 3 \mathrm{H}), 3.02(\mathrm{q}, J=7.1 \mathrm{~Hz}, 4 \mathrm{H})$, $1.37(\mathrm{t}, J=7.1 \mathrm{~Hz}, 3 \mathrm{H}), 0.96(\mathrm{t}, J=7.0 \mathrm{~Hz}, 6 \mathrm{H}) ;{ }^{13} \mathrm{C} \mathrm{NMR}\left(125 \mathrm{MHz}, \mathrm{CDCl}_{3}\right) \delta 166.7,163.6,157.3$, 156.6, 155.0, 148.6, 145.2, 132.6, 130.4, 130.1 (2C), 129.1, 128.2 (2C), 113.2, 111.2, 106.0, 61.5, 52.1, 45.8 (2C), 14.2, 12.0 (2C); HRMS (ESI) calcd for $\mathrm{C}_{24} \mathrm{H}_{25} \mathrm{NO}_{6} \mathrm{Na}(\mathrm{M}+\mathrm{Na})^{+} 446.1580$. Found 
446.1583; Anal. Calcd for $\mathrm{C}_{24} \mathrm{H}_{25} \mathrm{NO}_{6}: \mathrm{C}, 68.07 ; \mathrm{H}, 5.95 ; \mathrm{N}, 3.31$. Found: C, 67.78; H, 5.94; N, 3.30.

Ethyl 6-(4-(benzyloxycarbonyl)phenyl)-7-(diethylamino)-2-oxo-2 $H$-chromene-3- carboxylate 3k.

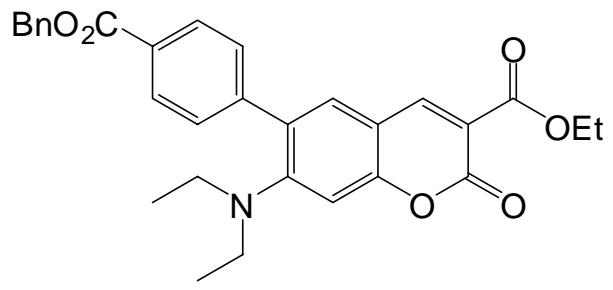

$3 \mathrm{k}$

Compound $3 \mathrm{k}$ was prepared from $2 \mathrm{k}$ according to the procedure described for $3 \mathrm{a}$. Yield $63 \%$ (pale yellow cubes). A small amount of this compound was recrystallized in $i-\operatorname{Pr}_{2} \mathrm{O}$ to give an analytical sample.

Mp $121-123^{\circ} \mathrm{C} ;{ }^{1} \mathrm{H}$ NMR $\left(500 \mathrm{MHz} \mathrm{CDCl}_{3}\right) \delta 8.44(\mathrm{~s}, 1 \mathrm{H}), 8.11(\mathrm{~d}, J=8.4 \mathrm{~Hz}, 2 \mathrm{H}), 7.52(\mathrm{~d}, J=8.5$ $\mathrm{Hz}, 2 \mathrm{H}), 7.46(\mathrm{~d}, J=7.0 \mathrm{~Hz}, 2 \mathrm{H}), 7.39(\mathrm{~m}, 2 \mathrm{H}), 7.34(\mathrm{~m}, 1 \mathrm{H}), 7.30(\mathrm{~s}, 1 \mathrm{H}), 6.86(\mathrm{~s}, 1 \mathrm{H}), 5.37(\mathrm{~s}, 2 \mathrm{H})$, 4.37 (q, $J=7.1 \mathrm{~Hz}, 2 \mathrm{H}), 3.01$ (q, $J=7.1 \mathrm{~Hz}, 4 \mathrm{H}), 1.37$ (t, $J=7.1 \mathrm{~Hz}, 3 \mathrm{H}), 0.95$ (t, $J=7.1 \mathrm{~Hz}, 6 \mathrm{H})$; ${ }^{13} \mathrm{C}$ NMR $\left(125 \mathrm{MHz}, \mathrm{CDCl}_{3}\right) \delta 166.1,163.6,157.4,156.7,155.0,148.6,145.3,136.0,132.6,130.4$, 130.2 (2C), 129.1, 128.6 (2C), 128.33, 128.27 (2C), 128.2 (2C), 113.3, 111.3, 106.1, 66.8, 61.5, 45.9 (2C), 14.3, 12.0 (2C); HRMS (ESI) calcd for $\mathrm{C}_{30} \mathrm{H}_{29} \mathrm{NO}_{6} \mathrm{Na}(\mathrm{M}+\mathrm{Na})^{+}$522.1893. Found 522.1888; Anal. Calcd for $\mathrm{C}_{30} \mathrm{H}_{29} \mathrm{NO}_{6} \cdot 1 / 8 \mathrm{H}_{2} \mathrm{O}: \mathrm{C}, 71.80 ; \mathrm{H}, 5.88 ; \mathrm{N}, 2.79$. Found: C, 71.70; H, 6.01; N, 2.81.

Ethyl 6-(4-amino-3-nitrophenyl)-7-(diethylamino)-2-oxo-2H-chromene-3-carboxylate 31.

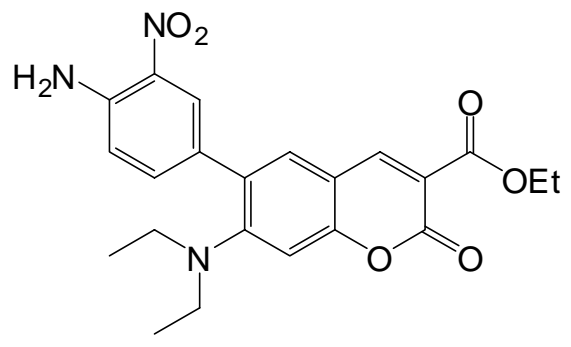

31

Compound 31 was prepared from 21 according to the procedure described for $3 \mathrm{a}$. Yield $62 \%$ (a pale orange powder). A small amount of this compound was recrystallized in EtOAc/hexane to give an analytical sample.

Mp 187-188 ${ }^{\circ} \mathrm{C} ;{ }^{1} \mathrm{H}$ NMR $\left(500 \mathrm{MHz}, \mathrm{CDCl}_{3}\right) \delta 8.44(\mathrm{~s}, 1 \mathrm{H}), 8.23(\mathrm{~d}, J=2.1 \mathrm{~Hz}, 1 \mathrm{H}), 7.52(\mathrm{dd}, J=8.6$, $2.1 \mathrm{~Hz}, 1 \mathrm{H}), 7.31(\mathrm{~s}, 1 \mathrm{H}), 6.89(\mathrm{~s}, 1 \mathrm{H}), 6.86(\mathrm{~d}, J=8.6 \mathrm{~Hz}, 1 \mathrm{H}), 6.21(\mathrm{br} \mathrm{s}, 2 \mathrm{H}), 4.37$ (q, $J=7.2 \mathrm{~Hz}$, $2 \mathrm{H}), 3.06$ (q, $J=7.1 \mathrm{~Hz}, 4 \mathrm{H}), 1.37$ (t, $J=7.1 \mathrm{~Hz}, 3 \mathrm{H}), 0.99$ (t, $J=7.1 \mathrm{~Hz}, 6 \mathrm{H}) ;{ }^{13} \mathrm{C} \mathrm{NMR}(125 \mathrm{MHz}$, $\left.\mathrm{CDCl}_{3}\right) \delta 163.5,157.4,156.2,155.2,148.6,144.0,135.8,132.1,132.1,129.8,128.9,125.0,119.2$, 113.3, 111.7, 106.7, 61.5, 45.6 (2C), 14.2, 11.9 (2C); HRMS (ESI) calcd for $\mathrm{C}_{22} \mathrm{H}_{22} \mathrm{~N}_{3} \mathrm{O}_{6}(\mathrm{M}-\mathrm{H})^{-}$ 
424.1509. Found 424.1513; Anal. Calcd for $\mathrm{C}_{22} \mathrm{H}_{23} \mathrm{~N}_{3} \mathrm{O}_{6}: \mathrm{C}, 62.11 ; \mathrm{H}, 5.45 ; \mathrm{N}, 9.88$. Found: C, $62.02 ; \mathrm{H}, 5.65 ; \mathrm{N}, 9.76$.

4-(7-(Diethylamino)-3-(ethoxycarbonyl)-2-oxo-2H-chromene-6-yl)benzoic acid 3m.

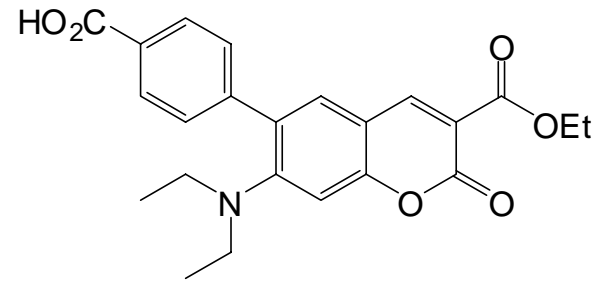

$3 \mathrm{~m}$

To a stirred solution of 3k (110.4 mg, $0.221 \mathrm{mmol})$ in MeOH/THF (2:1, v/v, $3 \mathrm{ml})$ was added 10\% $\mathrm{Pd} / \mathrm{C}(17.0 \mathrm{mg})$ at room temperature under an atmosphere of argon. $\mathrm{H}_{2}$ gass was introduced to the reaction mixture. After being stirred for $40 \mathrm{~min}$, the resultant mixture was filtered through Hyflo Super-Cel ${ }^{\circledR}$. The filtrate was concentrated. Purification of the residue by flash column chromatography (silica gel, 50\% EtOAc/hexane) gave $3 \mathrm{~m}(66.0 \mathrm{mg}, 73 \%)$ as a pale yellow powder. A small amount of this compound was recrystallized in EtOAc to give an analytical sample.

$\mathrm{Mp} 249-250^{\circ} \mathrm{C} ;{ }^{1} \mathrm{H} \mathrm{NMR}\left(500 \mathrm{MHz} \mathrm{CDCl}_{3}\right) \delta 11.06$ (br s, $\left.1 \mathrm{H}\right), 8.46(\mathrm{~s}, 1 \mathrm{H}), 8.15(\mathrm{~d}, J=8.3 \mathrm{~Hz}, 2 \mathrm{H})$, $7.56(\mathrm{~d}, J=8.3 \mathrm{~Hz}, 2 \mathrm{H}), 7.33(\mathrm{~s}, 1 \mathrm{H}), 6.86(\mathrm{~s}, 1 \mathrm{H}), 4.36(\mathrm{q}, J=7.1 \mathrm{~Hz}, 2 \mathrm{H}), 3.03(\mathrm{q}, J=7.1 \mathrm{~Hz}, 4 \mathrm{H})$, $1.36(\mathrm{t}, J=7.1 \mathrm{~Hz}, 3 \mathrm{H}), 0.96(\mathrm{t}, J=7.1 \mathrm{~Hz}, 6 \mathrm{H}) ;{ }^{33} \mathrm{C} \mathrm{NMR}\left(125 \mathrm{MHz}, \mathrm{CDCl}_{3}\right) \delta 170.9,163.6,157.4$, 156.7, 154.9, 148.6, 146.1, 132.7, 130.8 (2C), 130.3, 128.4 (2C), 128.1, 113.4, 111.4, 106.2, 61.6, 46.0 (2C), 14.3, 12.0 (2C); HRMS (ESI) calcd for $\mathrm{C}_{23} \mathrm{H}_{22} \mathrm{NO}_{6}(\mathrm{M}-\mathrm{H})^{-}$408.1447. Found 408.1450; Anal. Calcd for $\mathrm{C}_{23} \mathrm{H}_{23} \mathrm{NO}_{6} \cdot 1 / 4 \mathrm{H}_{2} \mathrm{O}: \mathrm{C}, 66.74 ; \mathrm{H}, 5.72 ; \mathrm{N}, 3.38$. Found: C, 66.98; H, 5.76; N, 3.35.

Ethyl 6-(3,4-diaminophenyl)-7-(diethylamino)-2-oxo-2 H-chromene-3-carboxylate 3n.

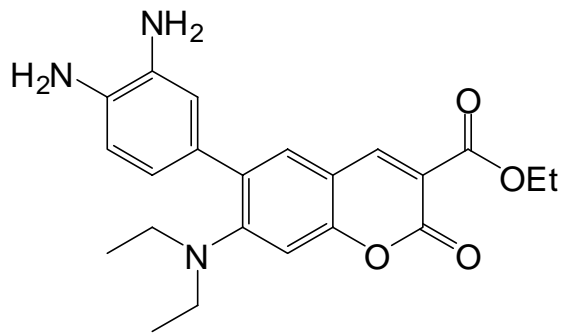

$3 \mathrm{n}$

To a stirred solution of 31 (120.9 mg, $0.284 \mathrm{mmol})$ in $\mathrm{MeOH}(2.5 \mathrm{ml})$ was added 10\% $\mathrm{Pd} / \mathrm{C}(39.1$ $\mathrm{mg}$ ) at room temperature under an atmosphere of argon. $\mathrm{H}_{2}$ gass was introduced to the reaction mixture. After being stirred for $2 \mathrm{~h}$, the resultant mixture was filtered through Hyflo Super-Cel ${ }^{\circledR}$. The filtrate was concentrated. Purification of the residue by flash column chromatography (silica gel, $\left.2.5 \% \mathrm{MeOH} / \mathrm{CHCl}_{3}\right)$ gave $3 \mathrm{n}(72.5 \mathrm{mg}, 65 \%)$ as an orange powder. A small amount of this 
compound was recrystallized in $\mathrm{MeOH} / i=\mathrm{Pr}_{2} \mathrm{O}$ to give an analytical sample.

Mp 185-186 ${ }^{\circ} \mathrm{C} ;{ }^{1} \mathrm{H}$ NMR (500 MHz, $\left.\mathrm{CDCl}_{3}\right) \delta 8.41$ (s, $\left.1 \mathrm{H}\right), 7.23(\mathrm{~s}, 1 \mathrm{H}), 6.82-6.75(\mathrm{~m}, 4 \mathrm{H}), 4.36$ (q, $J$ $=7.1 \mathrm{~Hz}, 2 \mathrm{H}), 3.68(\mathrm{br} \mathrm{s}, 4 \mathrm{H}), 3.07(\mathrm{q}, J=7.1 \mathrm{~Hz}, 4 \mathrm{H}), 1.37(\mathrm{t}, J=7.1 \mathrm{~Hz}, 3 \mathrm{H}), 0.96(\mathrm{t}, J=7.1 \mathrm{~Hz}$, 6H); ${ }^{13} \mathrm{C} \mathrm{NMR} \mathrm{(125} \mathrm{MHz,} \mathrm{CDCl}_{3}$ ) $\delta 163.9,157.9,156.1,155.3,149.0,134.7,134.0,132.5,132.4$, 131.5, 120.0, 116.9, 116.4, 112.0, 110.8, 105.2, 61.3, 45.5 (2C), 14.2, 12.1 (2C); HRMS (ESI) calcd for $\mathrm{C}_{22} \mathrm{H}_{24} \mathrm{~N}_{3} \mathrm{O}_{4}(\mathrm{M}-\mathrm{H})^{-}$394.1767. Found 394.1760; Anal. Calcd for $\mathrm{C}_{22} \mathrm{H}_{25} \mathrm{~N}_{3} \mathrm{O}_{4} \cdot 1 / 4 \mathrm{H}_{2} \mathrm{O}$ : C, 66.07; H, 6.43; N, 10.51. Found: C, 66.01; H, 6.27; N, 10.44 .

Ethyl 6-(1H-benzo[d][1,2,3] triazol-5-yl)-7-(diethylamino)-2-oxo-2 $H$-chromene-3- carboxylate 3o.

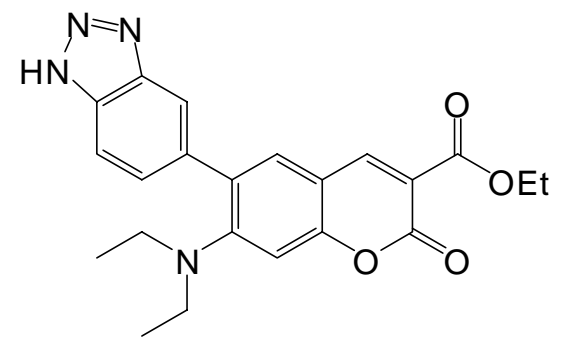

30

To a stirred solution of 3n (47.8 mg, $0.131 \mathrm{mmol})$ in $\mathrm{MeOH} / \mathrm{H}_{2} \mathrm{O}(1: 4, \mathrm{v} / \mathrm{v}, 2 \mathrm{ml})$ was added $\mathrm{AcOH}$ $(0.2 \mathrm{ml})$ and $\mathrm{NaNO}_{2}(21.4 \mathrm{mg} 0.310 \mathrm{mmol})$ at room temperature. After being stirred for $5 \mathrm{~min}$, the reaction mixture was diluted with EtOAc. The organic layer was washed with water and brine, dried, and concentrated. Purification of the residue by flash column chromatography (silica gel, $50 \%$ EtOAc/hexane) gave 3o (50.2 mg, 94\%) as a yellow powder. A small amount of this compound was recrystallized in $\mathrm{MeOH} / i-\mathrm{Pr}_{2} \mathrm{O}$ to give an analytical sample.

Mp 232-233 ${ }^{\circ} \mathrm{C} ;{ }^{1} \mathrm{H}$ NMR (500 $\left.\mathrm{MHz} \mathrm{CDCl}_{3}\right) \delta 8.51(\mathrm{~s}, 1 \mathrm{H}), 8.02(\mathrm{~s}, 1 \mathrm{H}), 7.98(\mathrm{~d}, J=8.6 \mathrm{~Hz}, 1 \mathrm{H})$, $7.55(\mathrm{dd}, J=8.6,1.0 \mathrm{~Hz}, 1 \mathrm{H}), 7.41(\mathrm{~s}, 1 \mathrm{H}), 6.91(\mathrm{~s}, 1 \mathrm{H}), 4.38(\mathrm{q}, J=7.1 \mathrm{~Hz}, 2 \mathrm{H}), 3.05$ (q, $J=7.1 \mathrm{~Hz}$, $4 \mathrm{H}), 1.36$ (t, $J=7.1 \mathrm{~Hz}, 3 \mathrm{H}), 0.96$ (t, $J=7.0 \mathrm{~Hz}, 6 \mathrm{H}) ;{ }^{13} \mathrm{C} \mathrm{NMR}\left(125 \mathrm{MHz}, \mathrm{CDCl}_{3}\right.$ ) $\delta 163.7,158.1$, 156.5, 155.2, 149.0, 139.3, 138.4, 138.3, 133.4, 131.1, 127.0, 115.5, 114.1, 112.5, 111.4, 106.0, 61.7, 52.2, 46.0 (2C), 14.3, 12.1 (2C);HRMS (ESI) calcd for $\mathrm{C}_{22} \mathrm{H}_{21} \mathrm{~N}_{4} \mathrm{O}_{4}(\mathrm{M}-\mathrm{H})^{-}$405.1563. Found 405.1564; Anal. Calcd for $\mathrm{C}_{22} \mathrm{H}_{22} \mathrm{~N}_{4} \mathrm{O}_{4}: \mathrm{C}, 65.01 ; \mathrm{H}, 5.46 ; \mathrm{N}, 13.78$. Found: C, 64.72; H, 5.60; N, 13.68. 
Table S1. Absrobance and Fluorescence maximum wavelengthes of 6-arylcoumarin compounds.

\begin{tabular}{|c|r|r|r|r|r|r|r|r|r|}
\hline Solvent & \multicolumn{3}{|c|}{$\mathrm{CH}_{3} \mathrm{CN}$} & \multicolumn{3}{c|}{$\mathrm{CH}_{3} \mathrm{OH}$} & \multicolumn{3}{|c|}{ Water } \\
\hline Comp. & Abs. Max. & Ex. Max. & Em. Max & Abs. Max. & Ex. Max. & Em. Max & Abs. Max. & Ex. Max. & Em. Max \\
\hline 3a & 410 & 407 & 462 & 415 & 407 & 462 & 431 & 401 & 465 \\
\hline 3b & 419 & n.d. & n.d. & 423 & n.d. & n.d. & 436 & n.d. & n.d. \\
\hline 3c & 411 & 403 & 471 & 416 & 410 & 477 & 431 & 410 & 480 \\
\hline 3d & 420 & n.d. & n.d. & 425 & n.d. & n.d. & 435 & n.d. & n.d. \\
\hline 3e & 401 & 392 & 444 & 404 & 397 & 449 & 425 & 408 & 457 \\
\hline 3f & 414 & 408 & 465 & 421 & 417 & 475 & 433 & 414 & 482 \\
\hline 3g & 412 & 406 & 466 & 418 & 414 & 472 & 433 & 418 & 484 \\
\hline 3h & 406 & 404 & 458 & 411 & 411 & 467 & 427 & 409 & 474 \\
\hline 3i & 403 & 399 & 454 & 406 & 404 & 458 & 427 & 400 & 464 \\
\hline 3j & 406 & 402 & 455 & 410 & 409 & 463 & 427 & 415 & 467 \\
\hline 31 & 409 & n.d. & n.d. & 412 & n.d. & n.d. & 428 & n.d. & n.d. \\
\hline 3m & 407 & 400 & 454 & 415 & 410 & 465 & 430 & 411 & 470 \\
\hline 3n & 420 & n.d. & n.d. & 425 & n.d. & n.d. & 437 & n.d. & n.d. \\
\hline 3o & 408 & 407 & 462 & 412 & 410 & 466 & 430 & 404 & 477 \\
\hline
\end{tabular}

Absorbance maximum wavelength and excitation, emission maximum wavelength of each compound $(3 \mu \mathrm{M})$ was measured in acetonitrile, methanol and water ( $1 \mathrm{mM}$ sodium phosphate buffer ( $\mathrm{pH}$ 7.4)). All solutions contained 0.3\% DMSO as a cosolvent. n.d.: no fluorescent peak was detected. 
1
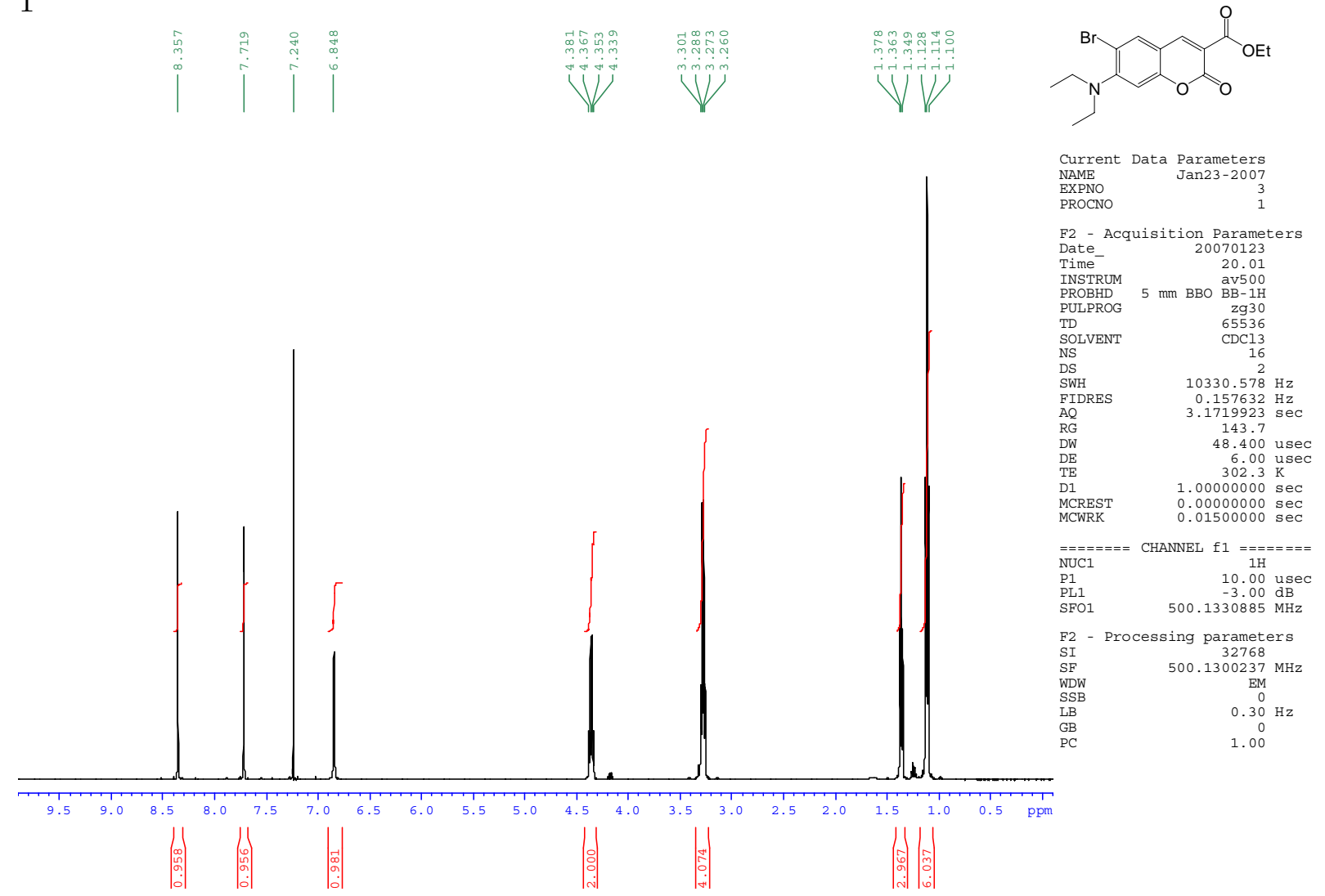

1

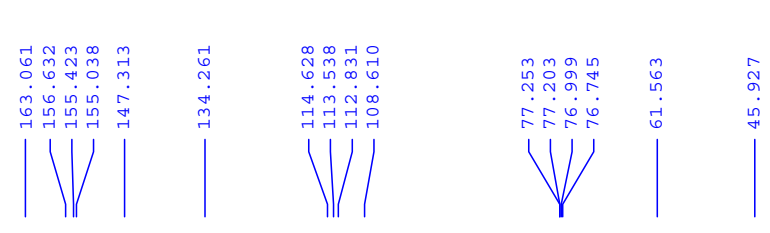

국

मे

$\mid$

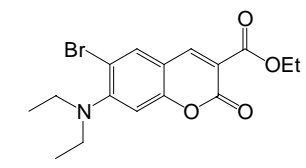

$\begin{array}{lr}\text { Current Data } & \text { Parameters } \\ \text { NAME } & \text { Jan23-2007 } \\ \text { EXPNO } & 4 \\ \text { PROCNO } & 1\end{array}$

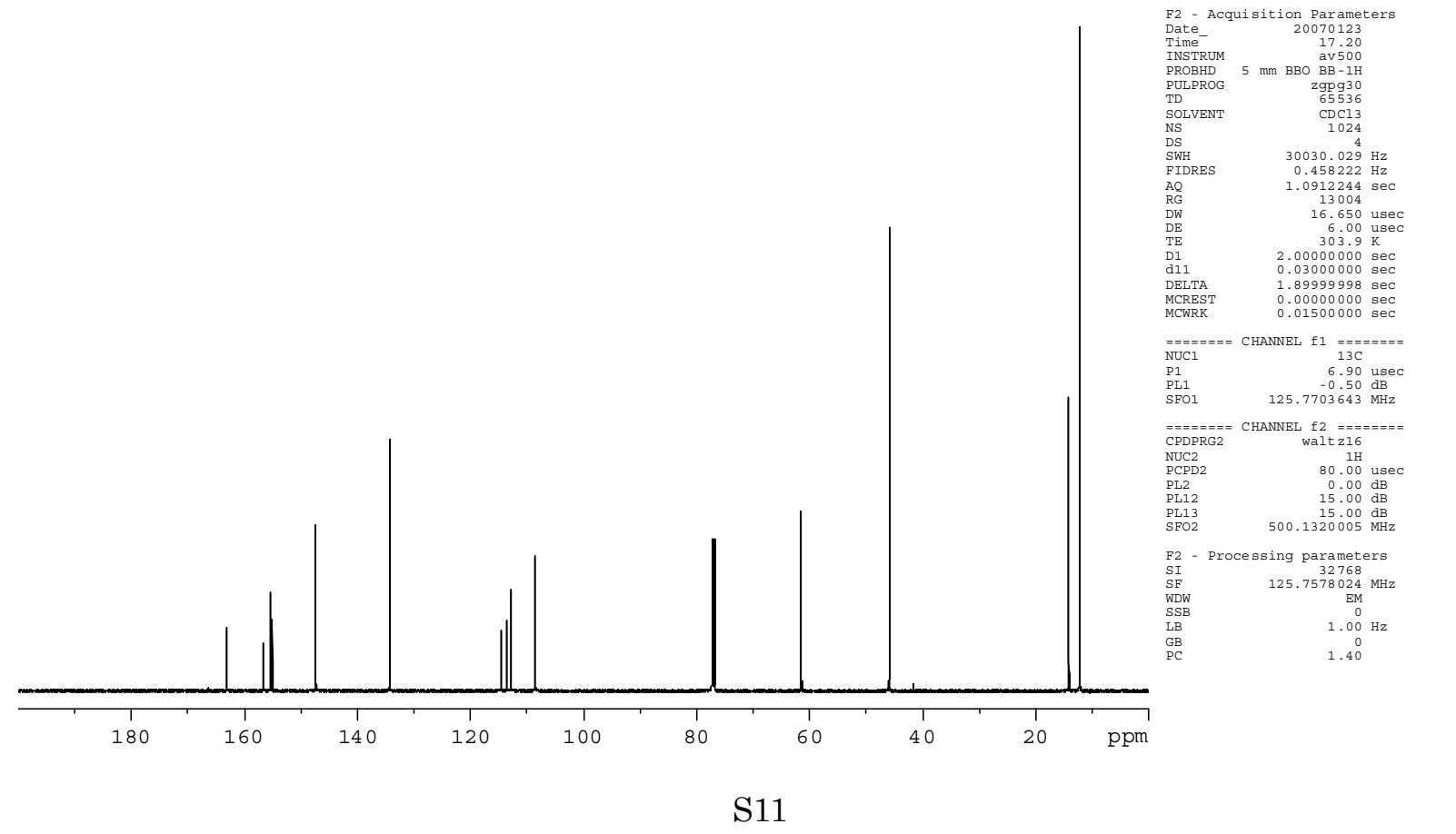


$3 a$
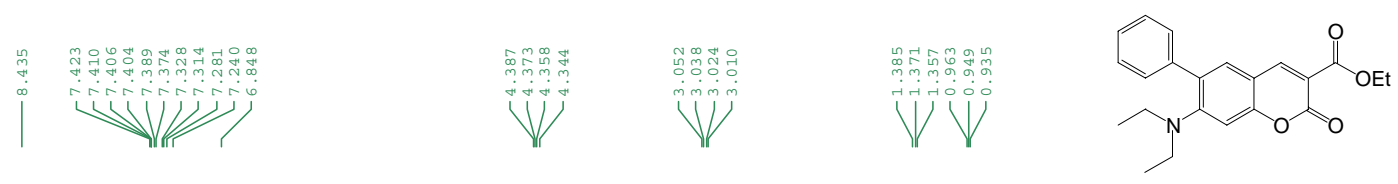

$\begin{array}{lr}\text { Current } & \text { Data } \\ \text { NAME } & \text { Janameters } \\ \text { EXPNO } & 4007 \\ \text { PROCN } & 4\end{array}$

PROCNO

an25-2007

F2 - Acquisition Parameters

Date

20070125

Time- 18.17

PROBHD $5 \mathrm{~mm}$ BBO BB-1H

PULPROG $\quad \mathrm{zg} 30$

TD

SOLVENT

NS

$\begin{array}{ll}2 \\ \text { SWH } & 10330.578 \mathrm{~Hz}\end{array}$

$\begin{array}{ll}\text { FIDRES } & 0.157632 \mathrm{~Hz} \\ \mathrm{AQ} & 3.1719923 \mathrm{sec}\end{array}$

RG

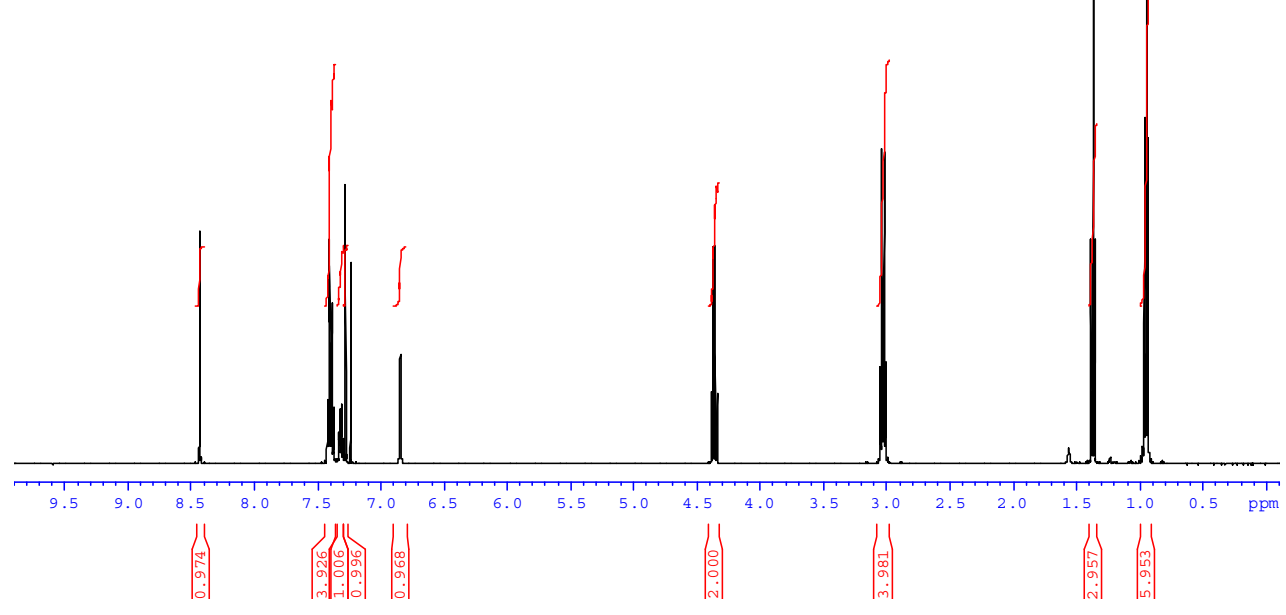

$3 a$

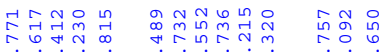

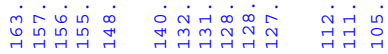

$|1| 1||||||$
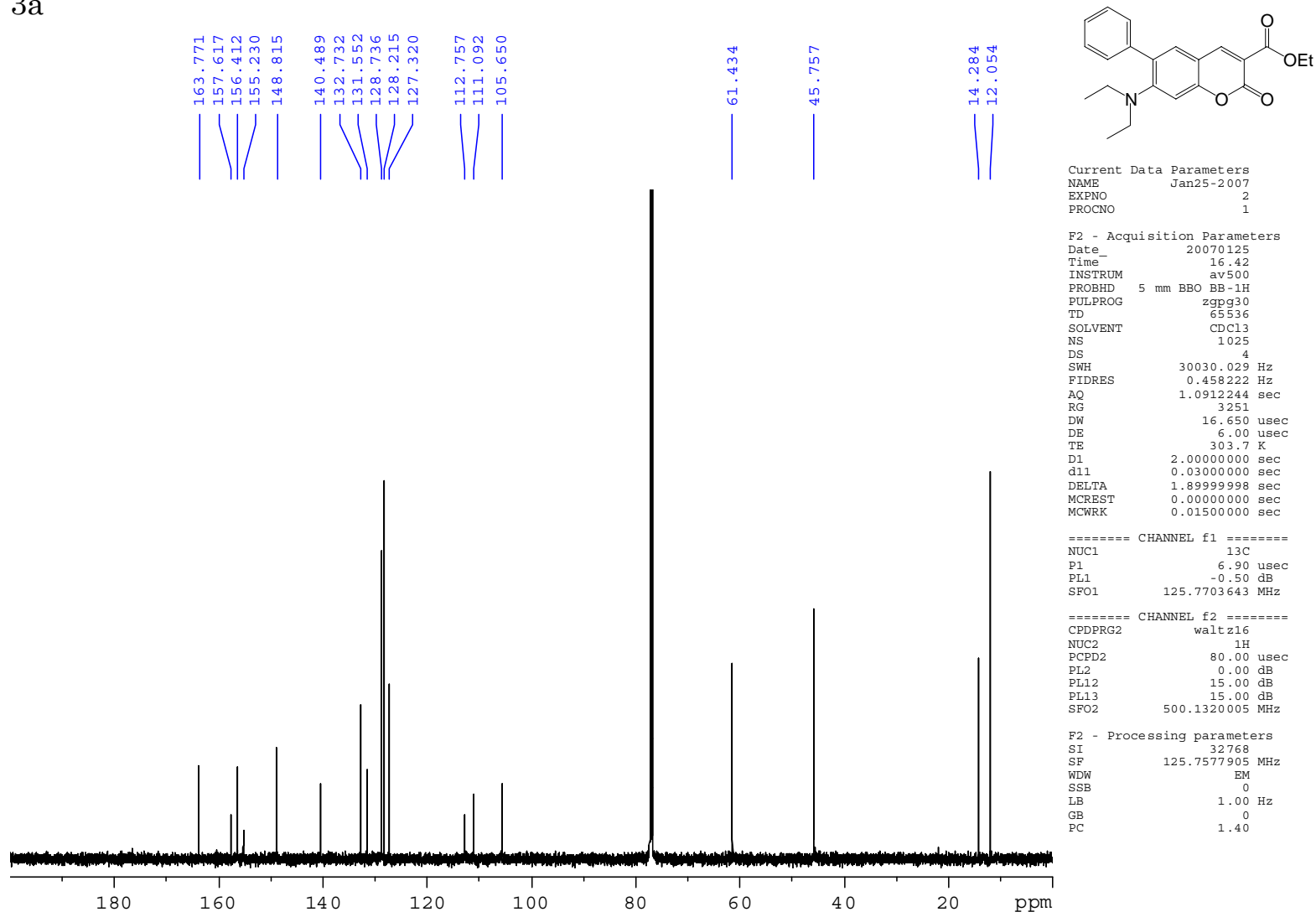

F2 - Acquisition Parameters

$\begin{array}{lr}\text { Date } & 20070125 \\ \text { Time } & 16.420\end{array}$

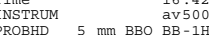

$\begin{array}{lr}\text { PGpg30 } \\ \text { TD } \\ \text { SOLVENT } & 65536 \\ & \mathrm{CDC13} \\ & 1025\end{array}$

$\begin{array}{lr}\text { NS } & 1025 \\ \text { DS } & 4 \\ \text { SWH } & 30030.029 \mathrm{~Hz}\end{array}$

$\begin{array}{lr}30030.029 \mathrm{~Hz} \\ \text { FIDRES } & 0.458222 \mathrm{~Hz} \\ & 1.0912244 \mathrm{sec}\end{array}$

RG
RW
DW
$16.650 \mathrm{usec}$

16.650 usec
DE
6.00
$\mathrm{DE}$

$\begin{array}{ll}2.0000000 \mathrm{sec} \\ \text { d11 } & 0.03000000 \mathrm{sec}\end{array}$

$\begin{array}{ll}\text { DETA } & 1.89999998 \mathrm{sec} \\ \text { MCRES } & 0.0000000 \mathrm{sec}\end{array}$

$=======$ CHANNEL f1 $========$
NUC1
13C

$\begin{array}{lr}\text { N1 } & 6.90 \text { usec } \\ \text { PL1 } & -0.50 \mathrm{~dB} \\ \text { SFO1 } & 125.770365 \mathrm{~d}\end{array}$

$=======$ CHANNEL $\begin{gathered}\mathrm{f} 2 \\ \text { walt } z 16\end{gathered} \mathrm{z}=====$
CPDPRG2

$\begin{array}{ll}\text { NUC2 } & 1 \mathrm{H} \\ \text { PCPD2 } & 80.00 \mathrm{usec} \\ \text { PL2 } & 0.00 \mathrm{~dB} \\ \text { PL12 } & 15.00 \mathrm{~dB}\end{array}$

$\begin{array}{lr}15.00 \mathrm{~dB} \\ \text { PL12 } & 15.00 \mathrm{~dB} \\ \text { PL13 } & 500.1320005 \mathrm{MH}\end{array}$

F2 - Processing parameters
SI
SF

$\begin{array}{lc}\text { SF } & 125.7577905 \text { MHz } \\ \text { WDW } & \text { EM } \\ \text { SSB } & 0\end{array}$

IB
GB
PC

$\underset{P C}{\mathrm{~GB}}$

60

ppm 
$3 b$
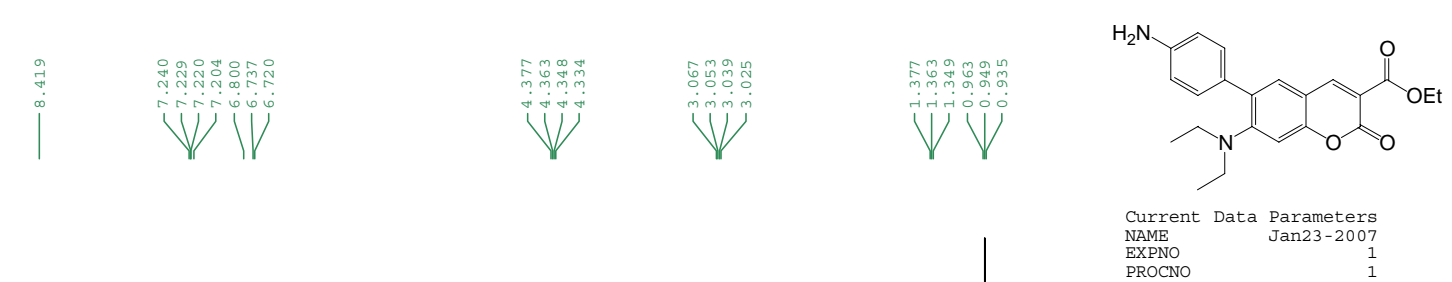

PROCNO

F2 - Acquisition Parameters

Date_ 20070123

$\begin{array}{ll}\text { Time } & 13.49 \\ \text { INSTRUM } & \text { av500 }\end{array}$

PROBHD $5 \mathrm{~mm}$ BBO BB-1H

$\begin{array}{lr}\text { PULPROG } & \mathrm{zg} 30 \\ \text { TD } & 65536\end{array}$

TD

NS
DS
SWH

$\begin{array}{lr}\text { DS } & 2 \\ \text { SWH } & 10330.578 \mathrm{~Hz} \\ \text { FIDRES } & 0.157632 \mathrm{~Hz}\end{array}$

$\begin{array}{ll}\text { AQ } & 0.157632 \mathrm{~Hz} \\ \text { RG } & 3.1719923 \mathrm{sec}\end{array}$

RG

$\mathrm{DW}$
$\mathrm{DE}$
$\mathrm{TE}$
$\mathrm{D} 1$

DCREST

228.1
48.400 usec 48.400 usec
6.00 usec

$\begin{array}{ll} & 1.00000000 \mathrm{sec} \\ \text { MCWRK } & 0.00000000 \mathrm{sec}\end{array}$

$=======$ CHANNEL $f 1$

$=======$ CHANNEL $\mathrm{f} 1 \mathrm{=}======$
NUC1 $1 \mathrm{H}$

$\begin{array}{lr}\text { P1 } & 10.00 \mathrm{usec} \\ \text { PL1 } & -3.00 \mathrm{~dB} \\ \text { SFO1 } & 500.1330885 \mathrm{MHz}\end{array}$

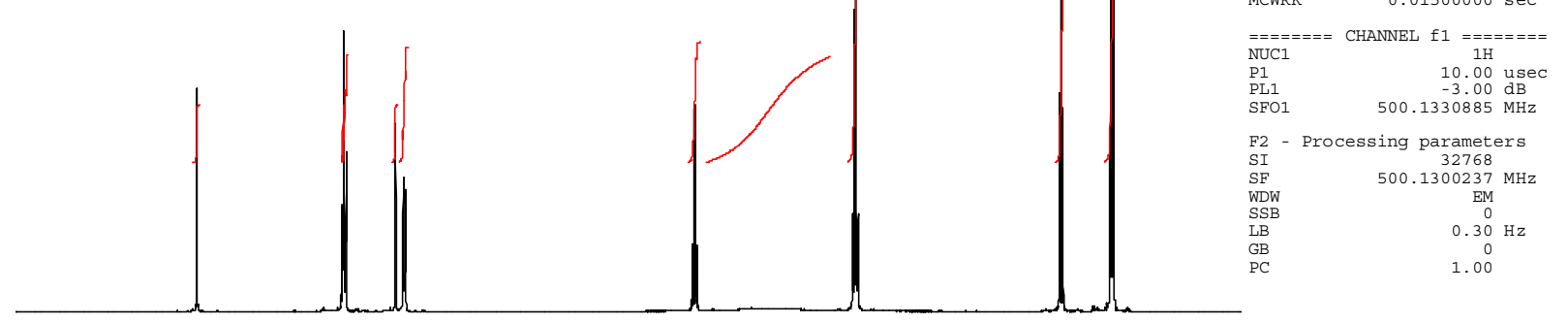

$\begin{array}{llllllllllllllllllllllllll}9.5 & 9.0 & 8.5 & 8.0 & 7.5 & 7.0 & 6.5 & 6.0 & 5.5 & 5.0 & 4.5 & 4.0 & 3.5 & 3.0 & 2.5 & 2.0 & 1.5 & 1.0 & 0.5 & \text { ppm }\end{array}$
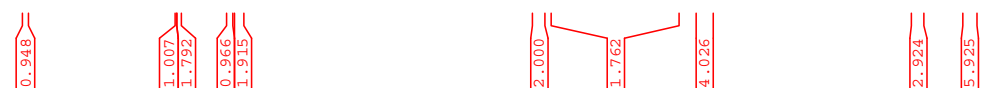

$3 b$

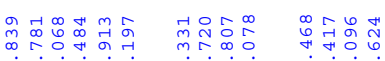

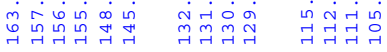

|W|| V| |V|

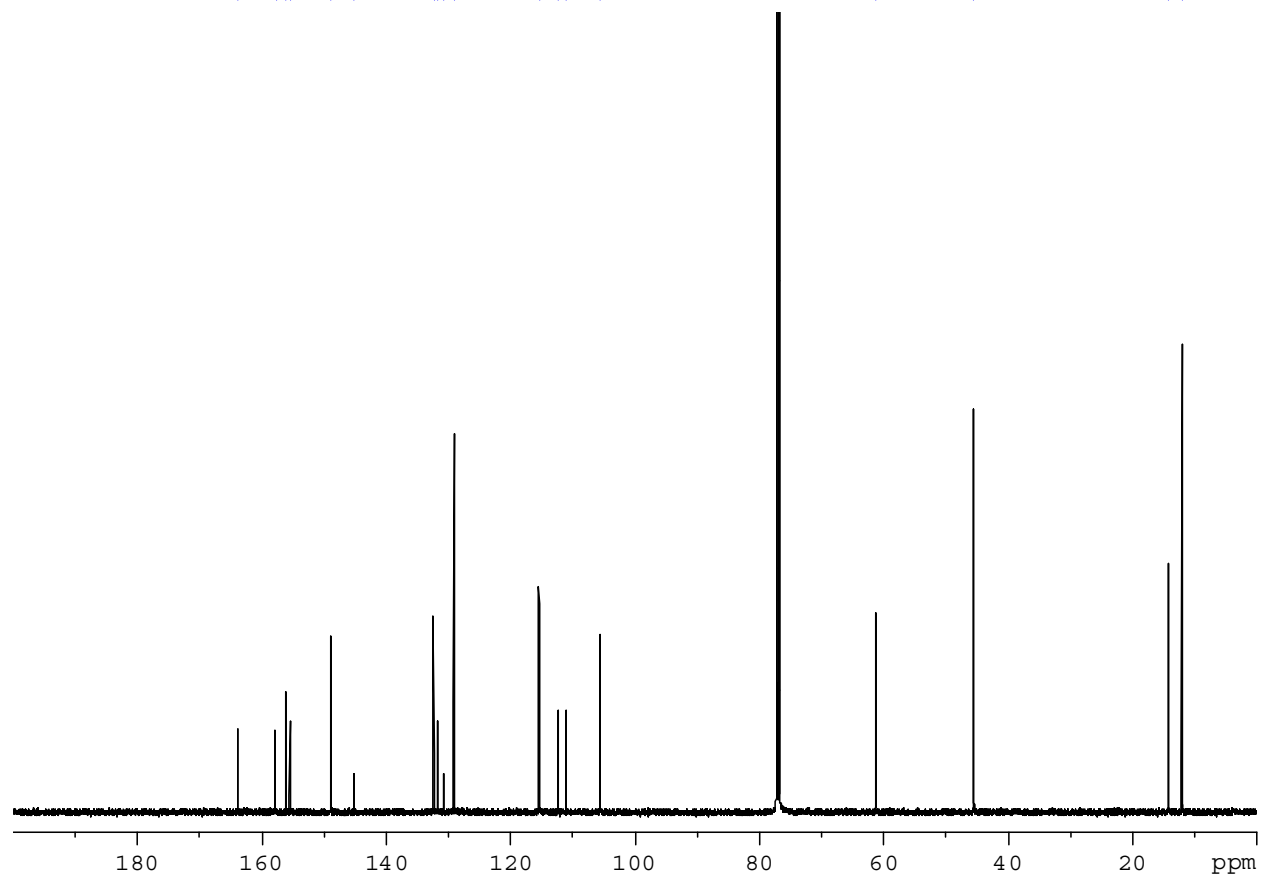

$\mathrm{H}_{2} \mathrm{~N}$
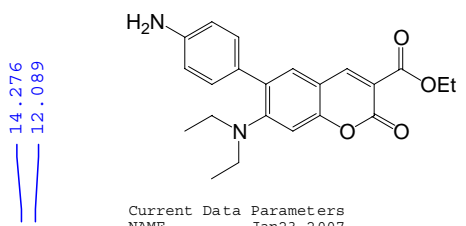

Current Data
NAME $\underset{\text { Janameters } 2007}{\text { NaPNO }}$

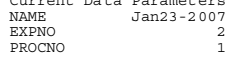

F2 -
Date_cuisition Parameters
20070123

$\begin{array}{lr}\text { Time } & 20070123 \\ \text { TNSTRUM } & 14.57\end{array}$

PROBHD
PUIPROG

$\begin{array}{lr}\text { PULPROG } & \text { zgpg30 } \\ \text { TD } & 65536 \\ \text { SOLVENT } & \text { CDC13 } \\ \text { IS } & 2048\end{array}$

$\begin{array}{lc}\text { DS } & 2048 \\ \text { SWH } & 30030.029 \mathrm{~Hz} \\ \text { PIDRES } & 0.458222 \mathrm{~Hz}\end{array}$

$\begin{array}{ll}0.458222 \mathrm{~Hz} \\ \mathrm{AQ} & 1.0912244 \mathrm{sec} \\ 3649.1 & 3649\end{array}$

$\begin{array}{ll}\text { RG } & 369.1 \\ \text { DW } & 16.650 \text { usec } \\ \text { DE } & 6.00 \text { used }\end{array}$

\begin{tabular}{l}
$16.00 \mathrm{u}$ \\
$\quad 304.0 \mathrm{~K}$ \\
\hline $\mathrm{E}$
\end{tabular}

$\begin{array}{ll}\text { D11 } & 2.00000000 \mathrm{sec} \\ \text { DELTA } & 0.03000000 \mathrm{sec}\end{array}$

MCREST $\quad 0.00000000 \mathrm{sec}$

$=======$ CHANNEL fi $=======$
NUC1
$13 \mathrm{C}$

$\begin{array}{lr}\text { P1 } & 6.90 \text { usec } \\ \text { PL1 } & -0.500 \mathrm{~dB} \\ \text { SFO1 } & 125.7705\end{array}$

$======8$ CHANNEL f $2=======$
walt $z 16$

$\begin{array}{lr}\text { NUC2 } & 1 \mathrm{H} \\ \text { PCPD2 } & 80.00 \mathrm{usec} \\ \text { PL2 } & 0.00 \mathrm{~dB} \\ \text { PL12 } & 15.00 \mathrm{~dB} \\ \text { PL13 } & 15.00 \mathrm{~dB} \\ \text { SFO2 } & 500.1320005 \mathrm{MHz} \\ \text { F2 - Processing parameters }\end{array}$

F2 - Processing parameters

$\begin{array}{lc}\text { SI } & 32768 \\ \text { SF } & 125.7577923 \mathrm{MHz} \\ \text { WDW } & \mathrm{EM} \\ \text { SSB } & 0 \\ \text { LB } & 1.00 \mathrm{~Hz} \\ \text { GB } & 0 \\ \text { PC } & 1.40\end{array}$ 
$3 \mathrm{c}$
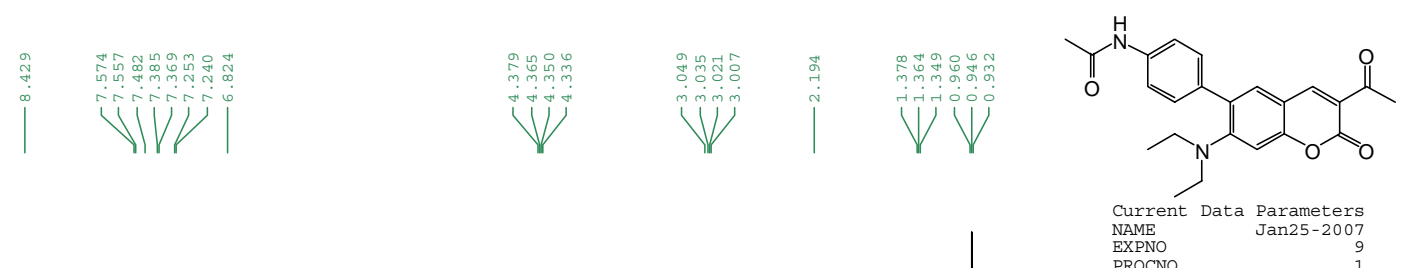

PROCNO

F2 - Acquisition Parameters

Date_ 20070125

$\begin{array}{ll}\text { Time } & - \\ \text { INSTRUM } & 23.14\end{array}$

PROBHD $5 \mathrm{~mm}$ BBO BB-1H

\begin{tabular}{lr} 
PULPROG & $\mathrm{zg} 30$ \\
\hline & 65536
\end{tabular}

TD

SOLVENT

DS
SWH

$\begin{array}{lr}2 \\ \text { SWH } & 10330.578 \mathrm{~Hz}\end{array}$

$\begin{array}{ll}\text { FIDRES } & 0.157632 \mathrm{~Hz} \\ \mathrm{AQ} & 3.1719923 \mathrm{sec}\end{array}$

AQ
RG
DW

$\begin{array}{lr}\text { DW } & 228.1 \\ \mathrm{DE} & 48.400 \mathrm{usec}\end{array}$

$\begin{array}{ll}\text { D1 } & 1.00000000 \mathrm{sec} \\ \text { MCREST } & 0.00000000 \mathrm{sec}\end{array}$

MCWRK $\quad 0.01500000 \mathrm{sec}$

$=======$ CHANNEL $\mathrm{f} 1 \mathrm{l}=======$

$\begin{array}{ll}\text { NUC1 } & 1 \mathrm{H} \\ \text { P1 } & 10.00 \mathrm{usec}\end{array}$

$\begin{array}{lr}\text { PL1 } & -3.00 \mathrm{~dB} \\ \text { SFO1 } & 500.1330885 \mathrm{MHz}\end{array}$
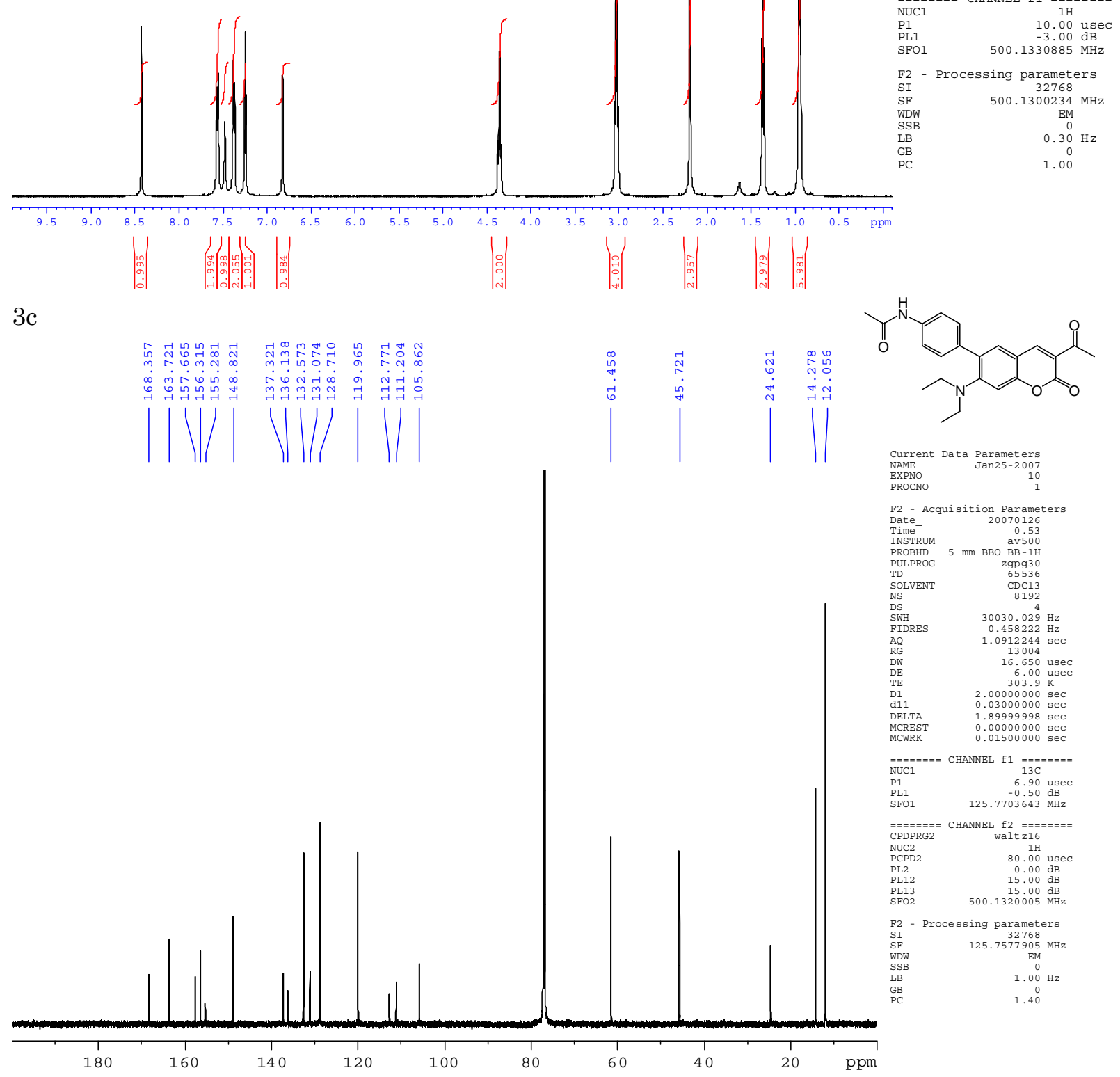
3d
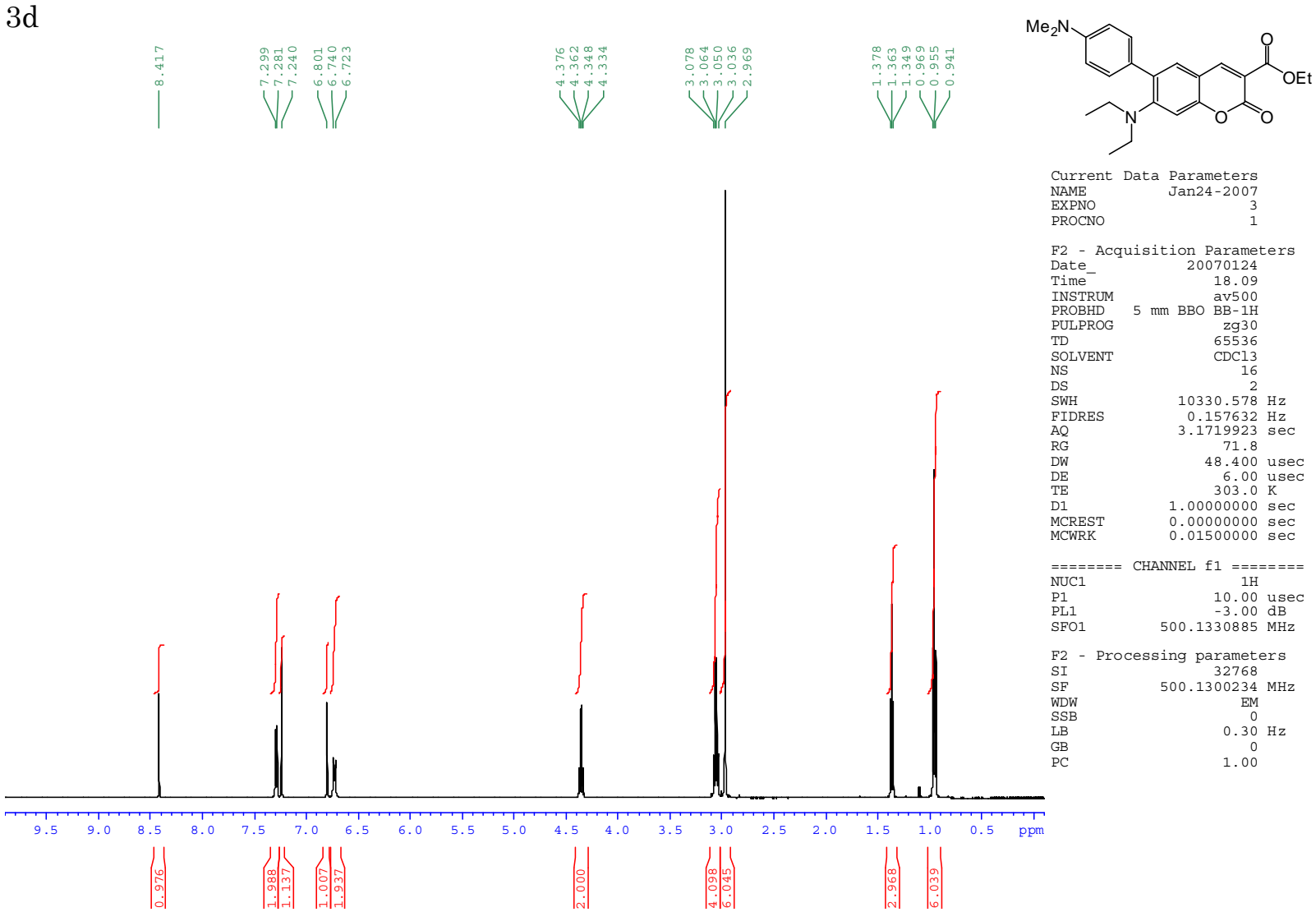

$3 d$
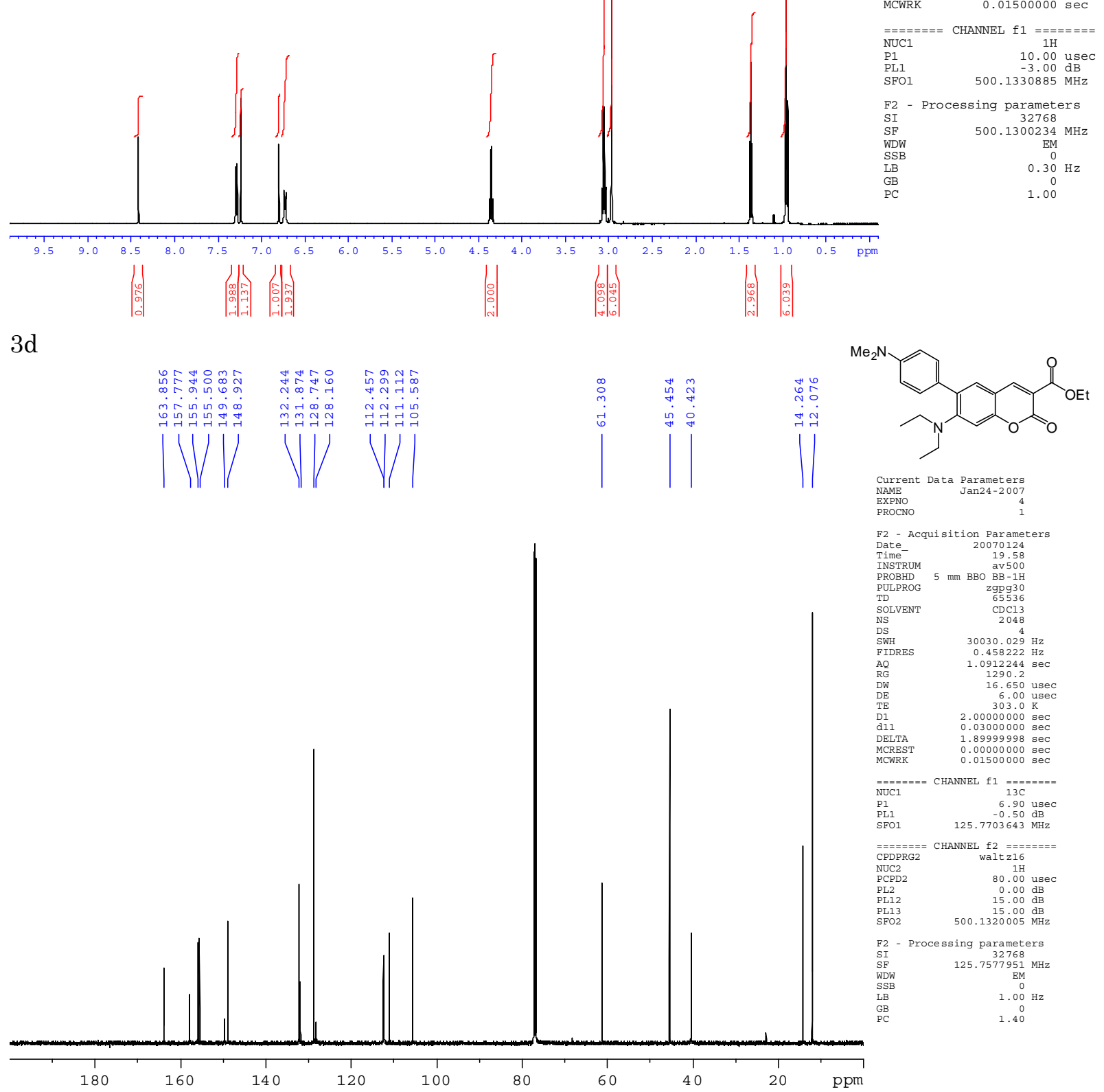
$3 e$
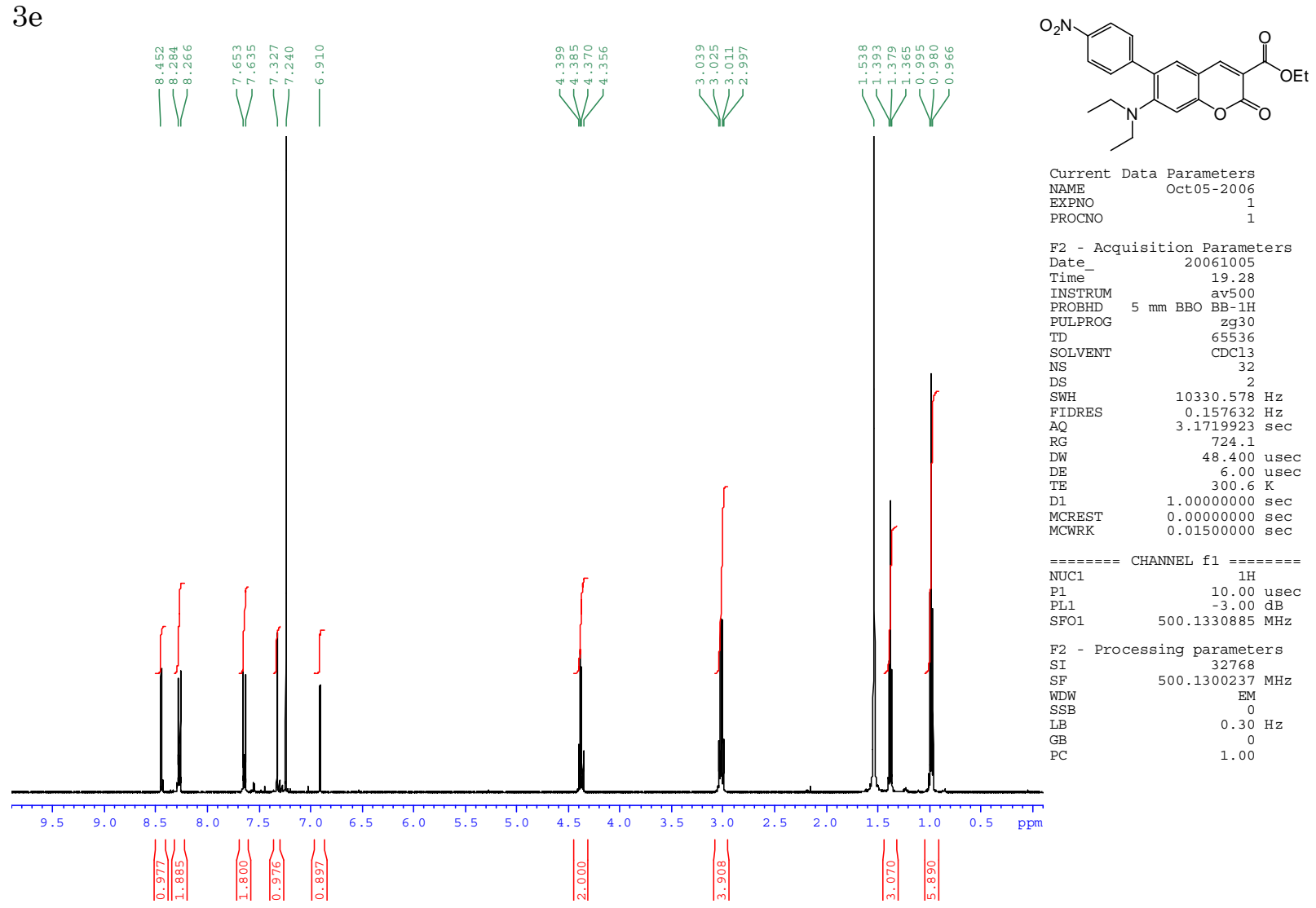

$3 e$
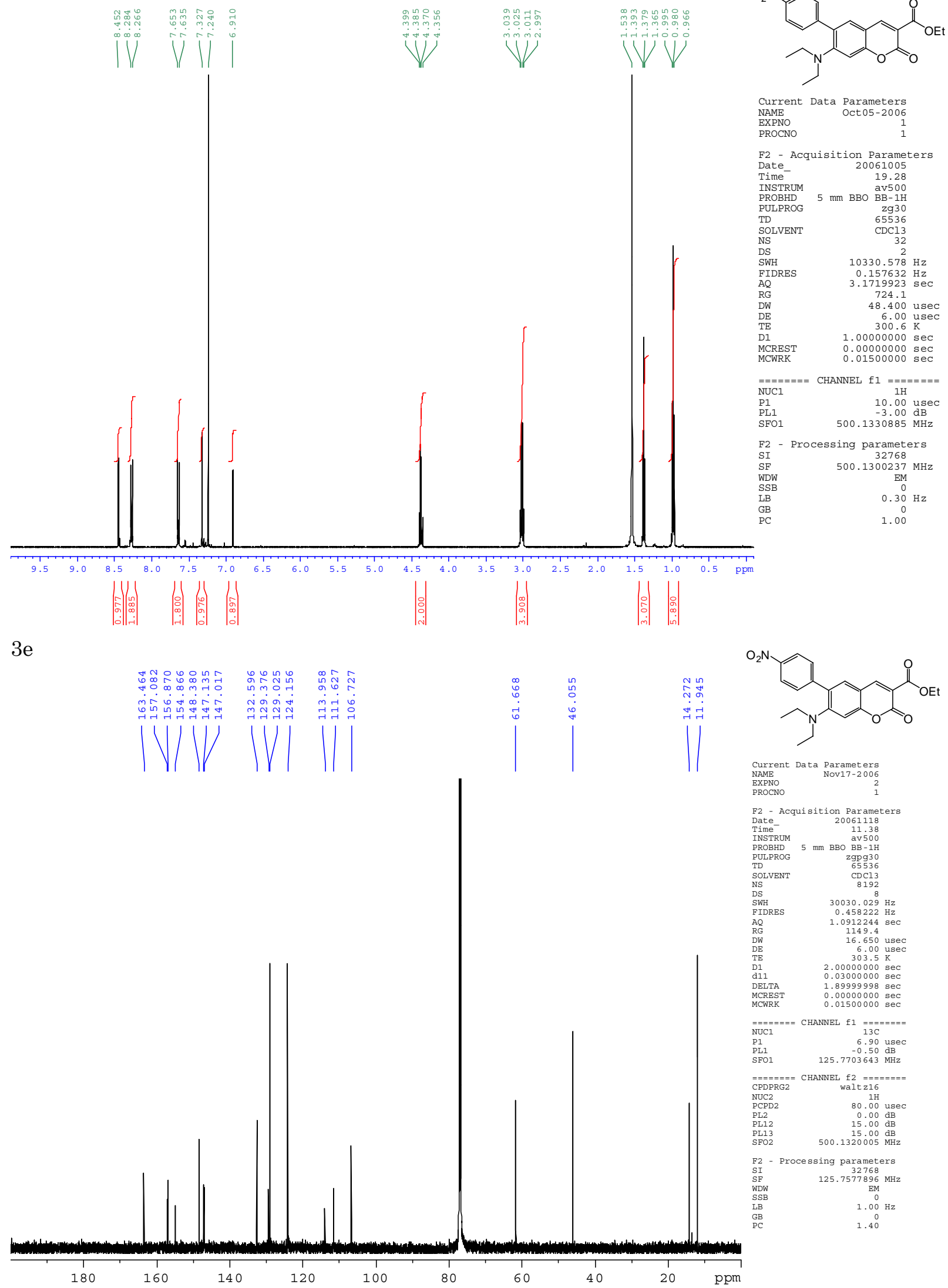

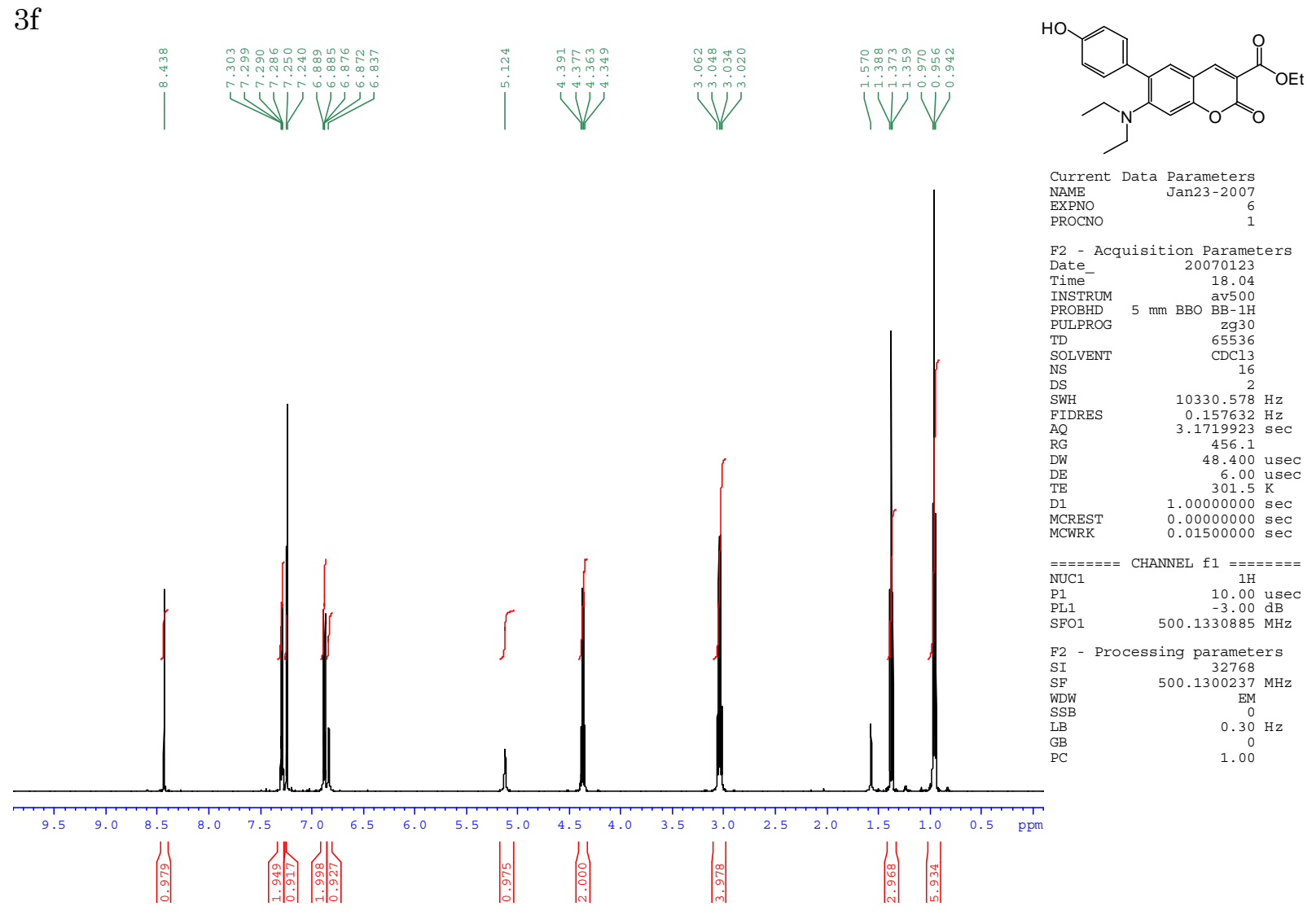

PROCNO

F2 - Acquisition Parameters

Date_ 20070123

$\begin{array}{ll}\text { Time } & 18.04 \\ \text { INSTRUM } & \end{array}$

PROBHD $5 \mathrm{~mm}$ BBO BB-1H

$\begin{array}{rr}\text { PULPROG } & \mathrm{zg} 30 \\ \text { TD } & 65536\end{array}$

TD

NS
DS
SWH

$\begin{array}{ll}2 & 2 \\ \text { SWH } & 10330.578 \mathrm{~Hz}\end{array}$

$\begin{array}{ll}\text { AQ } & 0.157632 \mathrm{~Hz} \\ \mathrm{R} & 3.1719923 \mathrm{sec}\end{array}$

RG

DE

6.00 usec
$301.5 \mathrm{~K}$

$\quad 1.00000000 \mathrm{sec}$

$\begin{array}{ll} & 0.00000000 \mathrm{sec} \\ \text { MCWRK } & 0.01500000 \mathrm{sec}\end{array}$

$=======$ CHANNEL $\mathrm{f} 1 \mathrm{l}=======$

$\begin{array}{ll}\text { P1 } & 1 \mathrm{H} \\ & 10.00 \mathrm{usec}\end{array}$

SFO1 $\quad 500.1330885 \mathrm{MHz}$

F2 - Processing parameters

$\begin{array}{lr}\mathrm{F} 2 & - \text { Processing parameters } \\ \mathrm{SI} & 32768 \\ \mathrm{SF} & 500.1300237 \mathrm{MHz}\end{array}$

$\begin{array}{lc}\text { SF } & 500.1300237 \mathrm{M} \\ \text { WDW } & \mathrm{EM} \\ \text { SSB } & 0 \\ \text { LB } & 0.30 \mathrm{~Hz} \\ \text { GB } & 0.00\end{array}$

$\begin{array}{lc}\mathrm{LB} & 0.30 \mathrm{~Hz} \\ \text { GB } & 0\end{array}$

$3 f$
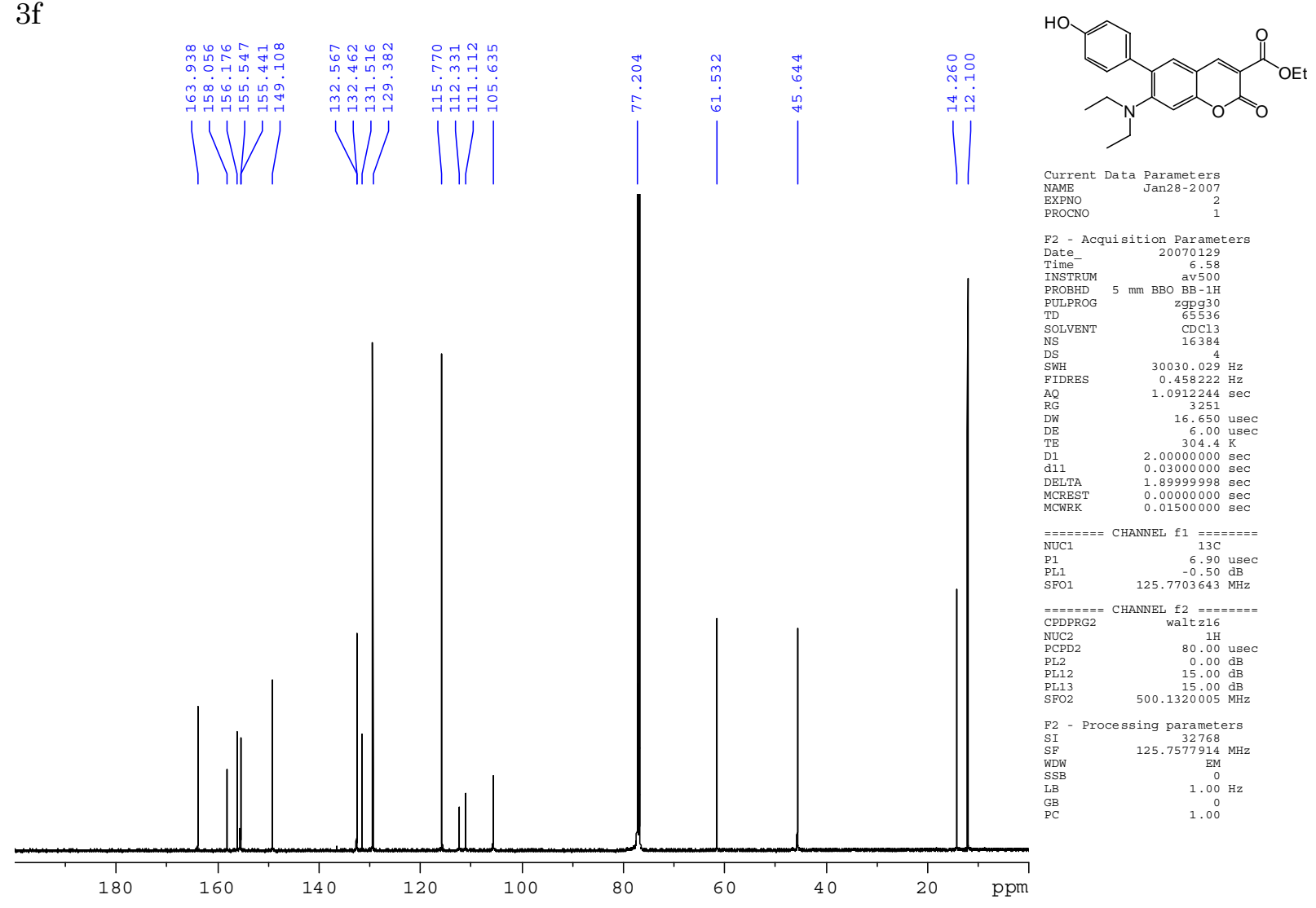

F2 - Acquisition Parameters
Date_
20070129

$\begin{array}{lr}\text { Time } & 20070129 \\ \text { TNSTRUM } & 6.58 \\ & \text { av500 }\end{array}$

PROBHD
PULPROG $\quad \begin{array}{r}\text { av } 500 \\ \text { BD } \\ \text { zgpg }-1 \mathrm{H} \\ \text { zglo }\end{array}$

$\begin{array}{ll}\text { SOLVENT } & 65536 \\ \text { CDC13 } & 16384\end{array}$

$\begin{array}{lc} & 16384 \\ \text { DS } & 4 \\ \text { SWH } & 30030.029 \mathrm{~Hz}\end{array}$

$\begin{array}{ll}\text { FWH } & 30030.029 \mathrm{~Hz} \\ \text { FIDRES } & 0.458222 \mathrm{~Hz} \\ \text { RQ } & 1.0912244 \mathrm{sec}\end{array}$

$\quad 3251$
$16.650 \mathrm{usec}$

$6.00 \mathrm{usec}$
$304.4 \mathrm{~K}$

$11 \quad 2.00000000 \mathrm{sec}$

$\begin{array}{ll}\text { DELTA } & 1.89999998 \mathrm{sec} \\ \text { MCREST } & 0.0000000 \mathrm{sec}\end{array}$

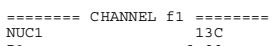

$\begin{array}{lr}\text { P1 } & 6.90 \text { usec } \\ \text { PL1 } & -0.50 \mathrm{~dB} \\ \text { SFO1 } & 125.7703643 \mathrm{MHz}\end{array}$

$=======$ CHANNEL $\mathrm{f} 2 \mathrm{f}=======$
walt $\mathrm{z} 16$

$\begin{array}{lc}\text { NUC2 } & 1 \mathrm{H} \\ \text { PCPD2 } & 80.00 \mathrm{usec} \\ \text { PL22 } & 0.00 \mathrm{~dB} \\ \text { PL12 } & 15.00 \mathrm{~dB}\end{array}$

$\begin{array}{lr}\text { PL12 } & 15.00 \mathrm{~dB} \\ \text { PL13 } & 15.00 \mathrm{~dB} \\ \text { SFO2 } & 500.1320005 \mathrm{MHz}\end{array}$

$\begin{array}{lr}\text { F2 } & \text { Processing parameters } \\ \text { SI } & 32768 \\ \text { SF } & 125.7577914 \mathrm{MHz} \\ \text { WDW } & \mathrm{EM} \\ \text { SSB } & 0 \\ \text { LB } & 1.00 \mathrm{~Hz}\end{array}$

$1.00 \mathrm{~Hz}$
1.00 
$3 \mathrm{~g}$
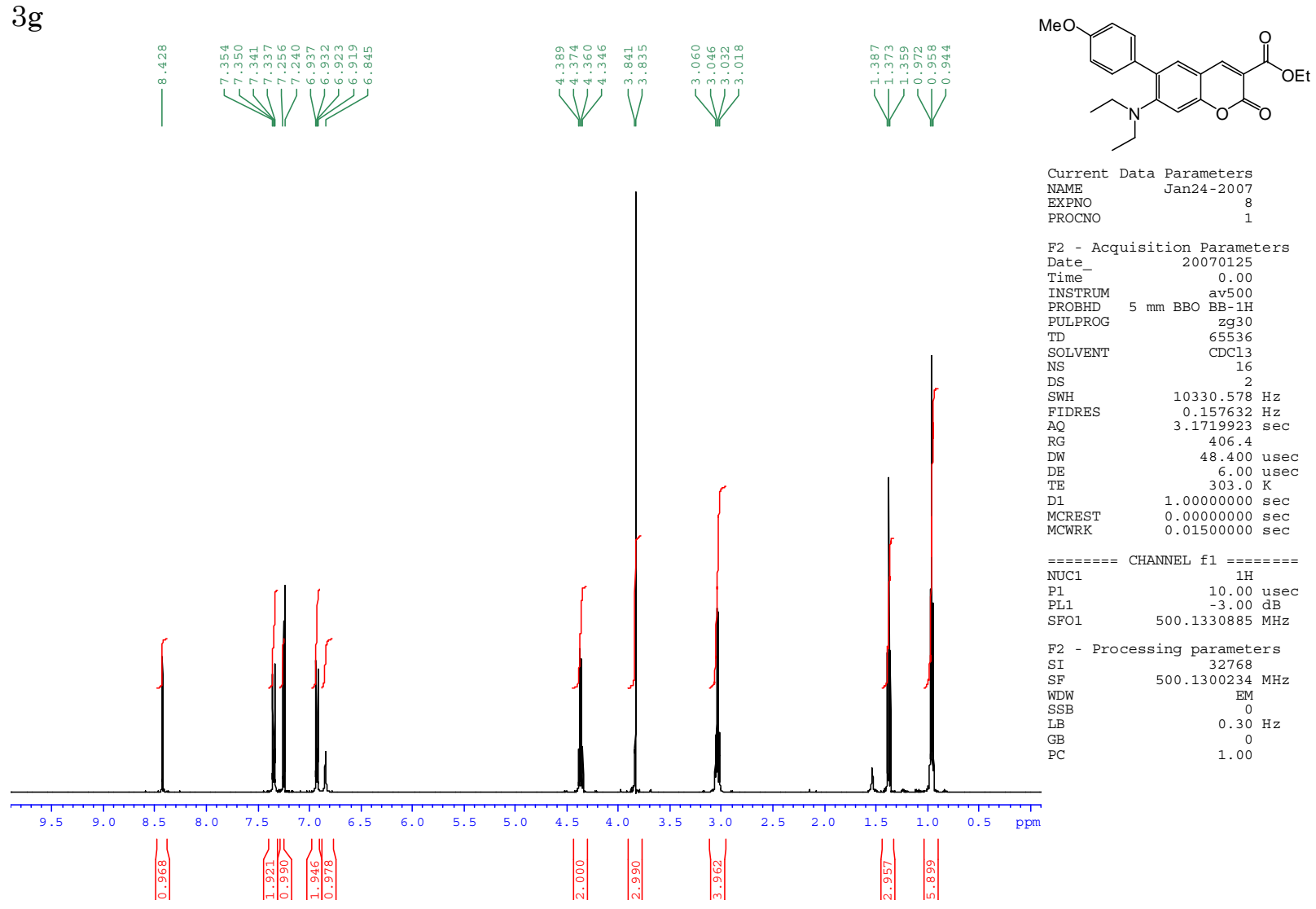

F2 - Acquisition Parameters

Date_r 20070125

Time-

PROBHD $5 \mathrm{~mm}$ BBO $\begin{aligned} & \text { av } 500 \\ & \text { BB- } 1 \mathrm{H}\end{aligned}$

$\begin{array}{rr}\text { PULPROG } & \mathrm{zg} 30 \\ 5 & 6556\end{array}$

TD

NS

DS

FIDRE
AQ

$\mathrm{RG}$
$\mathrm{DW}$
$\mathrm{DE}$

TE

MCRES

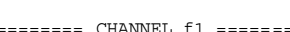

$z=== \pm==$ CHANNEL $\mathrm{fl}== \pm=====$
$1 \mathrm{H}$
$10.00 \mathrm{usec}$

$\begin{array}{lr}\text { P1 } & 10.00 \text { usec } \\ \text { PL1 } & -3.00 \mathrm{~dB} \\ \text { SFO1 } & 500.1330885 \mathrm{MHz}\end{array}$

$\begin{array}{lr}\text { F2 } & \text { - Processing parameters } \\ \text { SI } & 32768 \\ \text { SF } & 500.1300234 \mathrm{MHz}\end{array}$

$\begin{array}{lr}\text { SI } & 32768 \\ \text { WFW } & 500.1300234 \mathrm{MHz}\end{array}$

$\begin{array}{lc}\text { WDW } & \text { EM } \\ \text { SSB } & 0 \\ \text { LB } & 0.30 \mathrm{~Hz} \\ \text { GB } & 0 \\ \text { PC } & 1.00\end{array}$

$3 \mathrm{~g}$

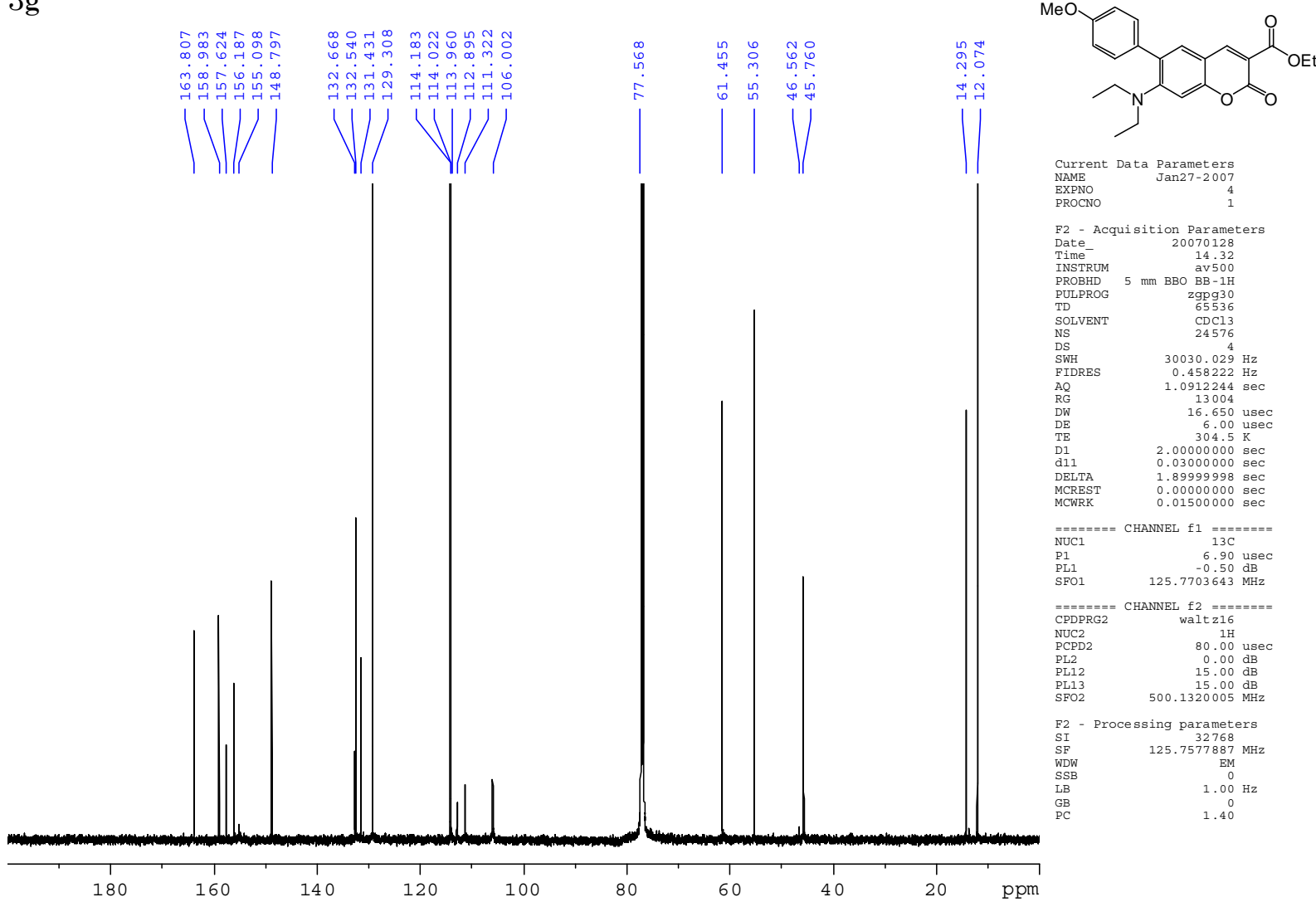


$3 \mathrm{~h}$
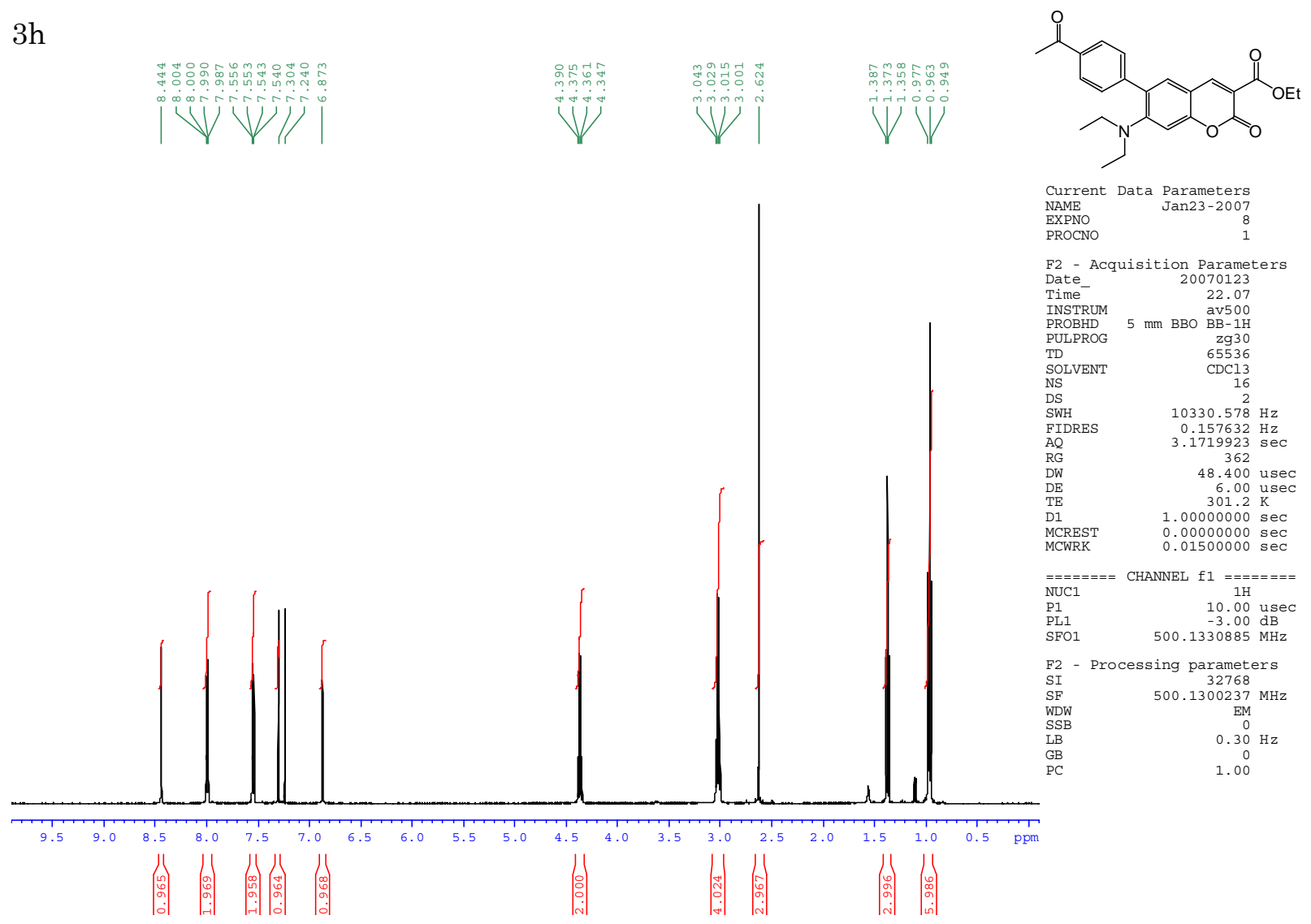

$3 \mathrm{~h}$

$\begin{array}{ll}\text { Current } & \text { Data } \\ \text { NAME } & \text { Jan23-2007 } \\ \text { JXPNO } & \end{array}$

PROCNO

F2 - Acquisition Parameters

Date_ 20070123

$\begin{array}{lr}22.07 \\ \text { INSTRUM } & \text { av500 }\end{array}$

PROBHD $5 \mathrm{~mm}$ BBO BB-1H

$\begin{array}{lr}\text { PULPROG } & \mathrm{zg} 30 \\ & 65536\end{array}$

TD

$\begin{array}{lr}\text { DS } & 2 \\ \text { SWH } & 10330.578 \mathrm{~Hz}\end{array}$

$\begin{array}{ll}\text { FIDRES } & 0.157632 \mathrm{~Hz} \\ \text { AQ } & 3.1719923 \mathrm{sec}\end{array}$

DW

D1

$====-2=$ CHANNET $f 1$

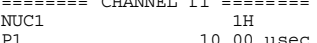

$\begin{array}{lr}\text { P1 } & 10.00 \mathrm{use} \\ \text { PL1 } & -3.00 \mathrm{~dB} \\ \text { SFO1 } & 500.1330885 \mathrm{MHz}\end{array}$

$\begin{array}{lr}\text { F2 } & \text { - Processing parameters } \\ \text { SI } & 32768 \\ \text { SF } & 500.1300237 \mathrm{MHz}\end{array}$

$\begin{array}{lc}\text { SF } & 500.1300237 \\ \text { WDW } & \text { EM } \\ \text { SSB } & 0 \\ \text { LB } & 0.30 \mathrm{~Hz}\end{array}$

LB
PC

PC
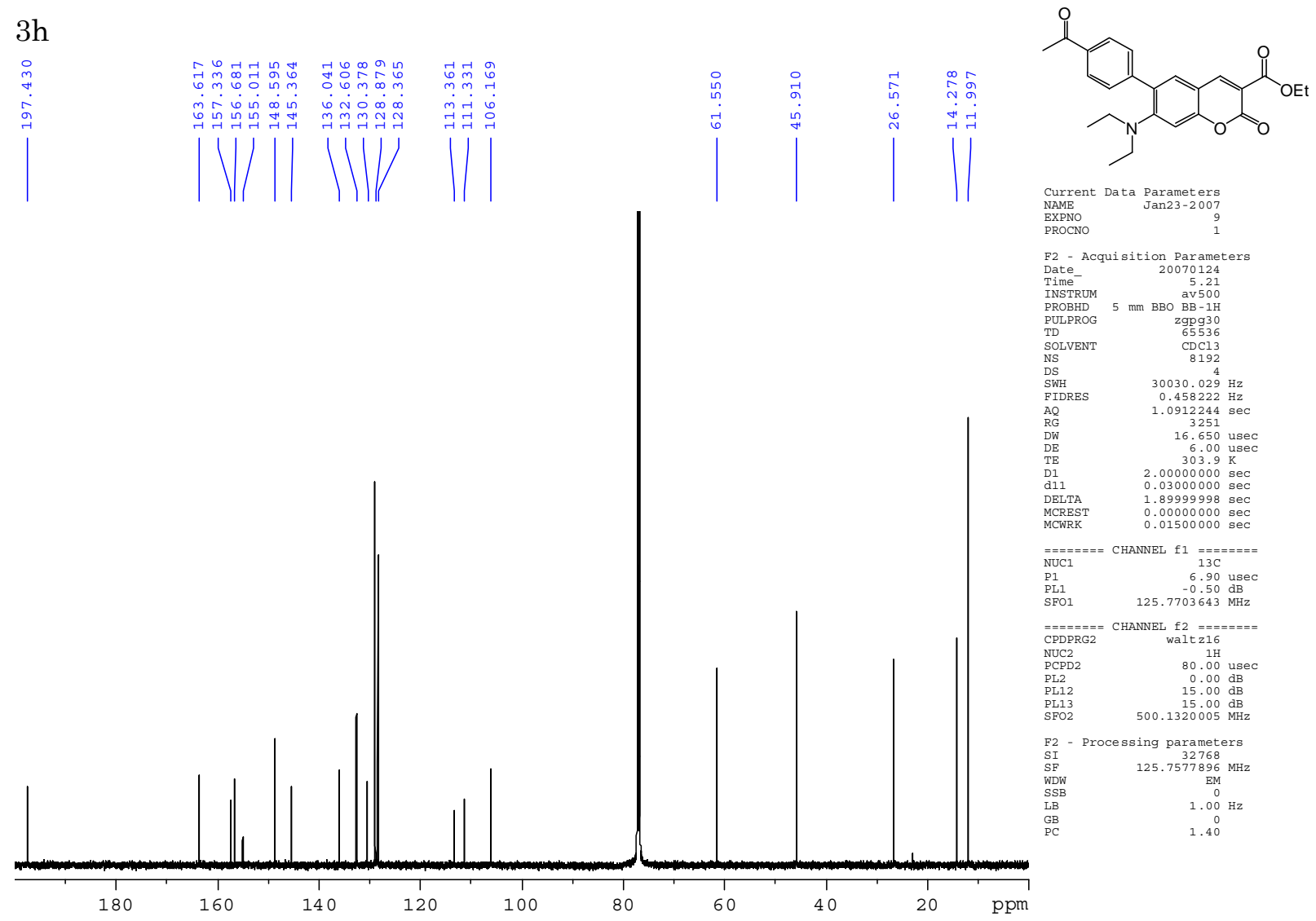

F2 - Acquisition Parameter
Date_- 20070124

TI
IN
PR
P
T
S
NS
D
S
P
A
A
RA
D
D
T
D
d
D

av 500
PROBHD $5 \mathrm{~mm}$ BBO BB-1H

zggp30
TL
OLVROG
CDC13
8192

$\begin{array}{lr}\text { NS } & \text { CDCl3 } \\ \text { NS } & 8192 \\ \text { SWH } & 40030.029 \mathrm{~Hz}\end{array}$

$0.4512244 \mathrm{~Hz}$
3251
$\mathrm{sec}$

3251
16.650 usec
6.000 usec

$3.00003 .9 \mathrm{~K}$
0.030000000
$\mathrm{sec}$

$\begin{array}{ll}\text { DELTA } & 1.89999998 \mathrm{sec} \\ \text { CREST } & 0.00000000 \mathrm{sec}\end{array}$

$0.01500000 \mathrm{sec}$

$=======$ CHANNEL $\mathrm{f} 1 \quad=======$
NUC1
$13 \mathrm{C}$

$\begin{array}{lr}\text { P1 } & 6.90 \text { usec } \\ \text { PL1 } & -0.50 \mathrm{~dB} \\ \text { SFO1 } & 125.7703643 \mathrm{MHz}\end{array}$

$=======$ CHANNEL $\mathrm{f} 2 \mathrm{f}======$
walt $\mathrm{z} 16$

$\begin{array}{ll}\text { NUC2 } & 1 \mathrm{H} \\ \text { PCPD2 } & 80.00 \mathrm{usec} \\ \text { PL2 } & 0.00 \mathrm{~dB} \\ \text { PL12 } & 15.00 \mathrm{~dB}\end{array}$

$\begin{array}{lr}15.00 \mathrm{~dB} \\ \text { PL12 } & 15.00 \mathrm{~dB} \\ \text { PL13 } & 500.1320005 \mathrm{MH}\end{array}$

F2 - Processing parameters

$\begin{array}{lc}\text { SF } & 125.7577896 \mathrm{MHz} \\ \text { WDW } & \mathrm{EM} \\ \text { WSB } & 0 \\ \text { SB } & 1.00 \mathrm{~Hz} \\ \text { GB } & 0 \\ \text { GC } & 1.40\end{array}$ 
$3 \mathrm{i}$

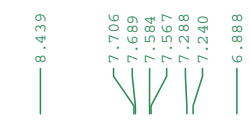

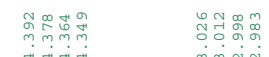 \\ $\bigcup^{\dot{m i n} \text { jij }^{j}}$}
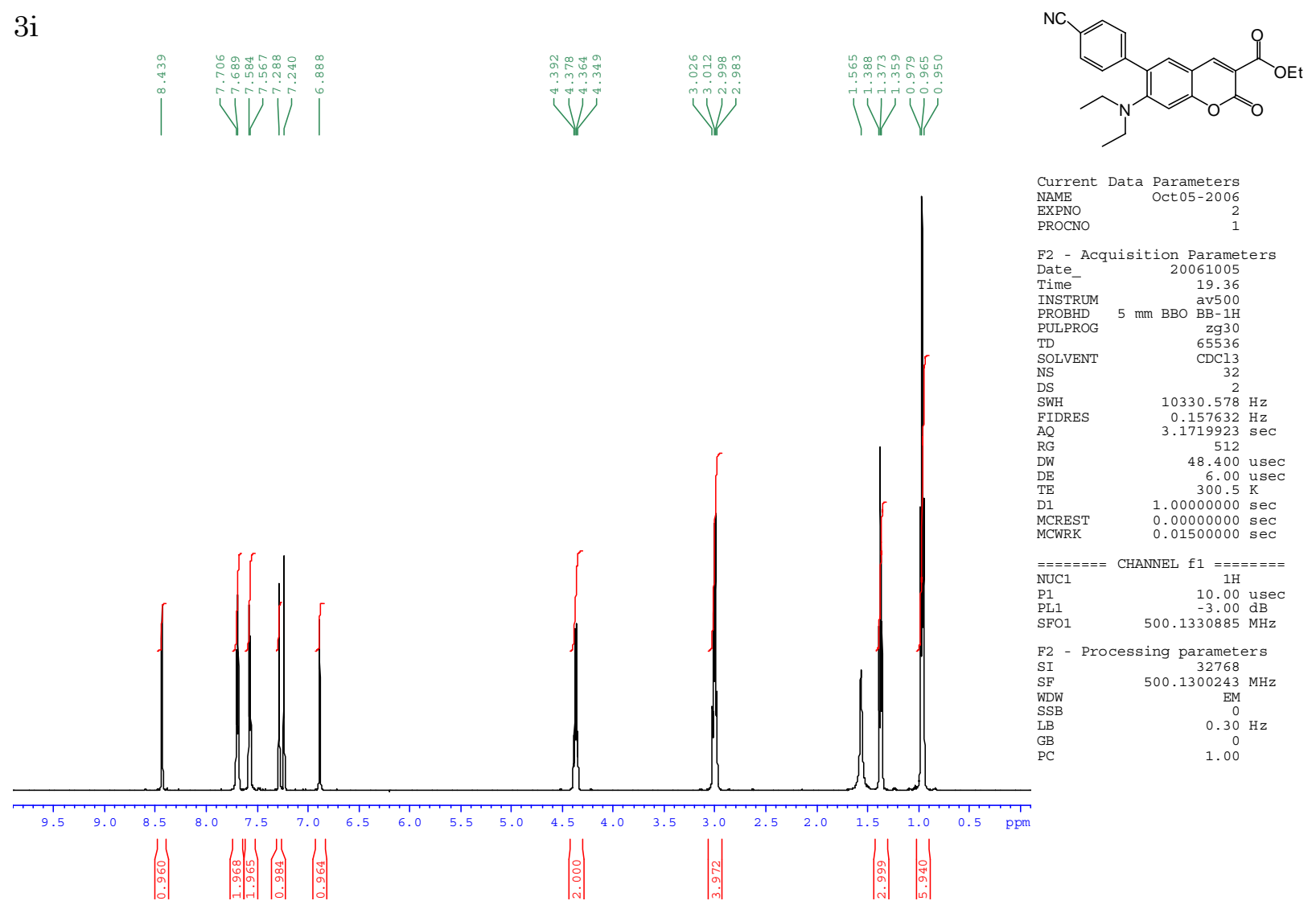

$3 \mathrm{i}$
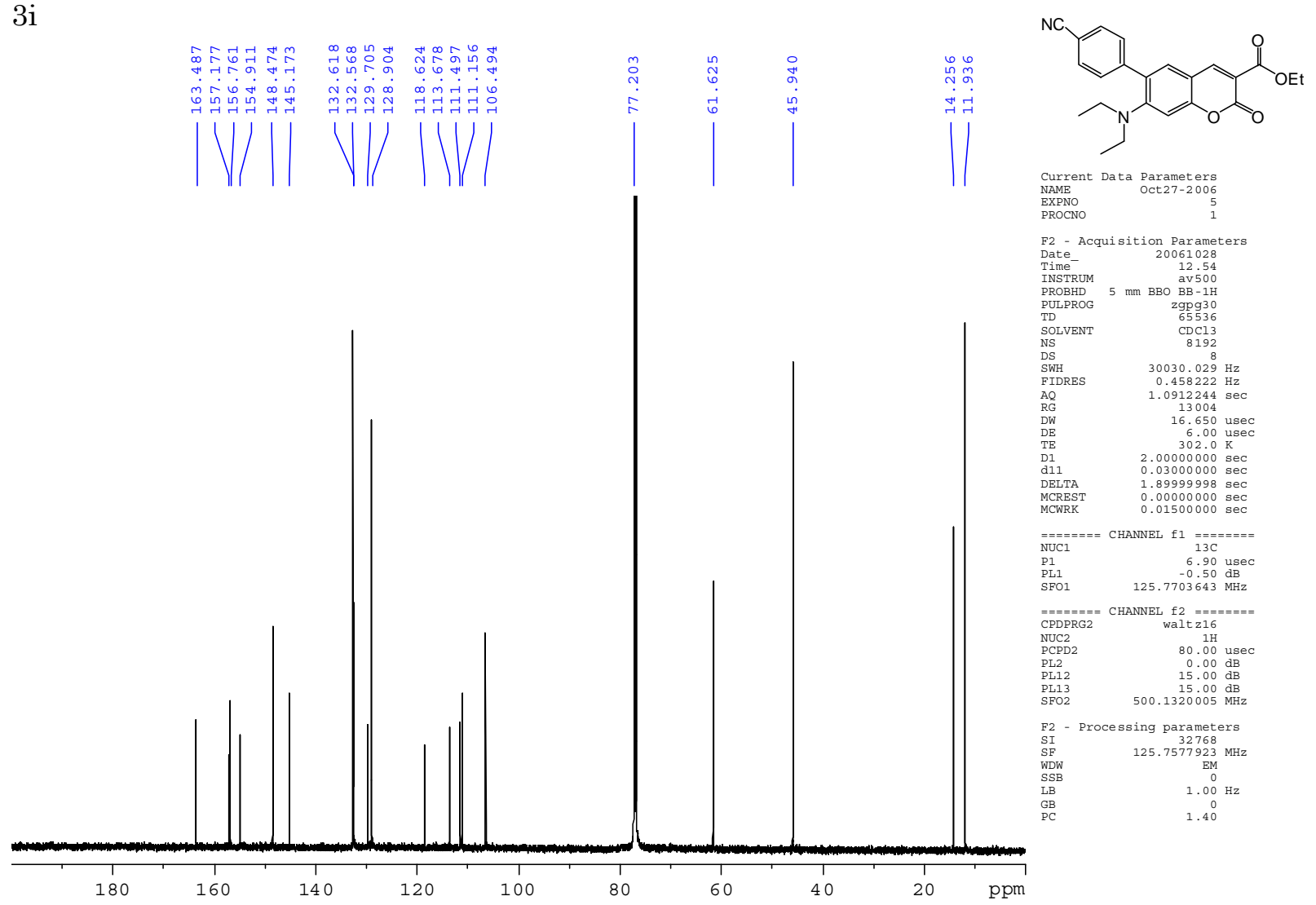
$3 \mathrm{j}$
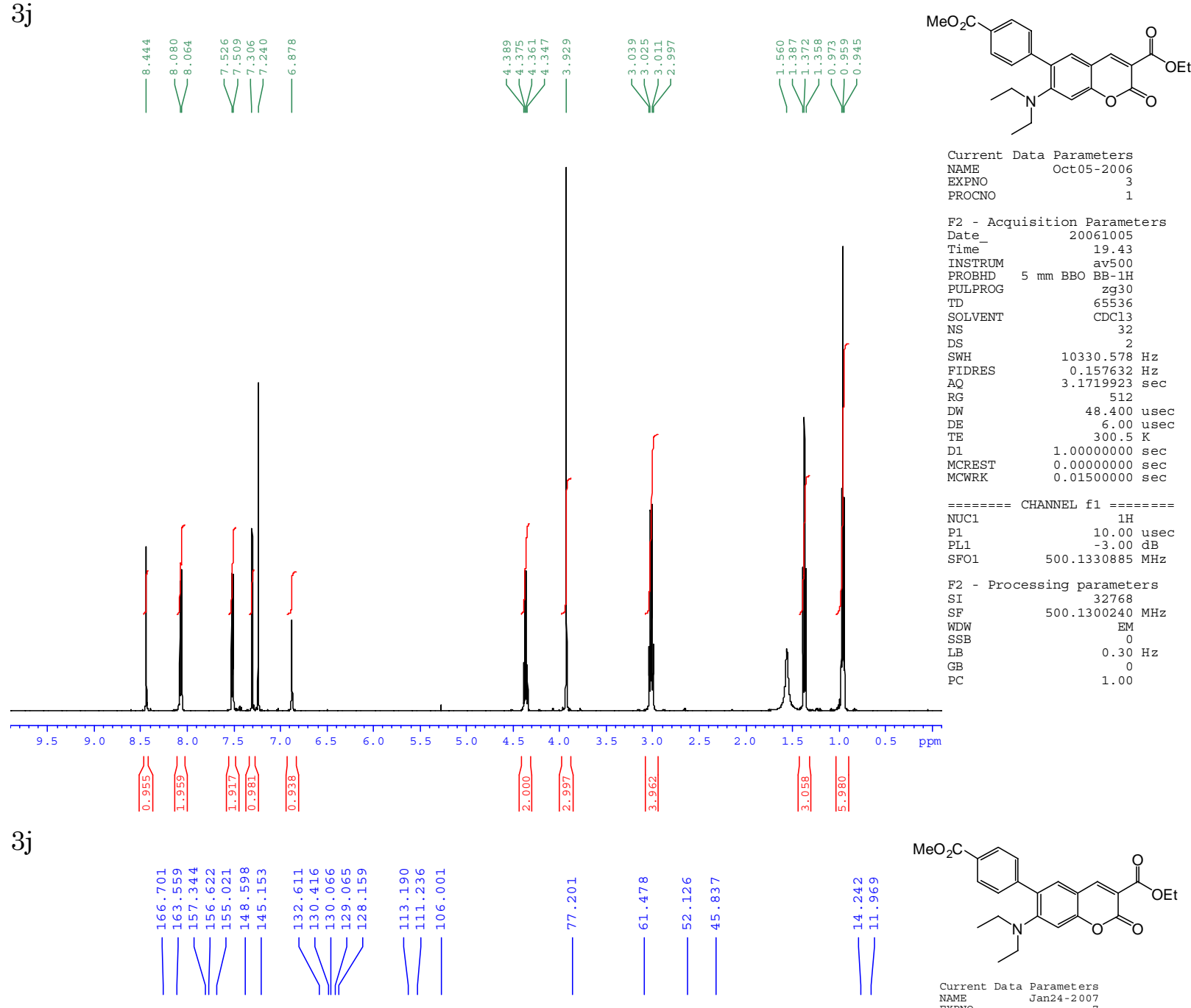

$\begin{array}{ll}\text { Current Data Parameters } \\ \text { NAME } & \text { Oct05-2006 }\end{array}$ NAME
EXPNO
PROCNO Oct $05-2006$
3

F2 - Acquisition Parameters Date__ 20061005 Time 19.43 PROBHD $5 \mathrm{~mm}$ BBO BB-1H PULPROG $\quad \mathrm{zg} 30$

TD

SOLVENT NS
SWH

SWH $\quad 10330.578 \mathrm{~Hz}$

$\begin{array}{ll}\text { FIDRES } & 0.157632 \mathrm{~Hz} \\ \text { AO } & 3.1719923 \mathrm{sec}\end{array}$

RG

$\mathrm{DW}$
$\mathrm{DE}$
$\mathrm{TE}$

D1

MCREST

512
48.400 usec 48.400 usec
6.00 usec
$300.5 \mathrm{~K}$

$1.00000000 \mathrm{sec}$

$0.00000000 \mathrm{sec}$
$0.01500000 \mathrm{sec}$

$=======$ CHANNEL $\mathrm{f} 1 \mathrm{l}========$

$\begin{array}{cc}======= & \text { CHANNEL } \\ \text { NUC1 } & 10.00 \text { usec }\end{array}$

$\begin{array}{lr}\text { P1 } & 10.00 \text { usec } \\ \text { PL1 } & -3.00 \mathrm{~dB}\end{array}$

SFO1 $500.1330885 \mathrm{MHz}$

F2

- Processing parameters

SF $\quad 500.1300240 \mathrm{MHz}$

$\begin{array}{lc}\text { WDW } & \text { EM } \\ \text { SSB } & 0 \\ \text { LB } & 0.30 \mathrm{~Hz}\end{array}$

$\begin{array}{lc}\text { LB } & 0.30 \mathrm{H} \\ \text { GB } & 0 \\ \text { PC } & 1.00\end{array}$

3j
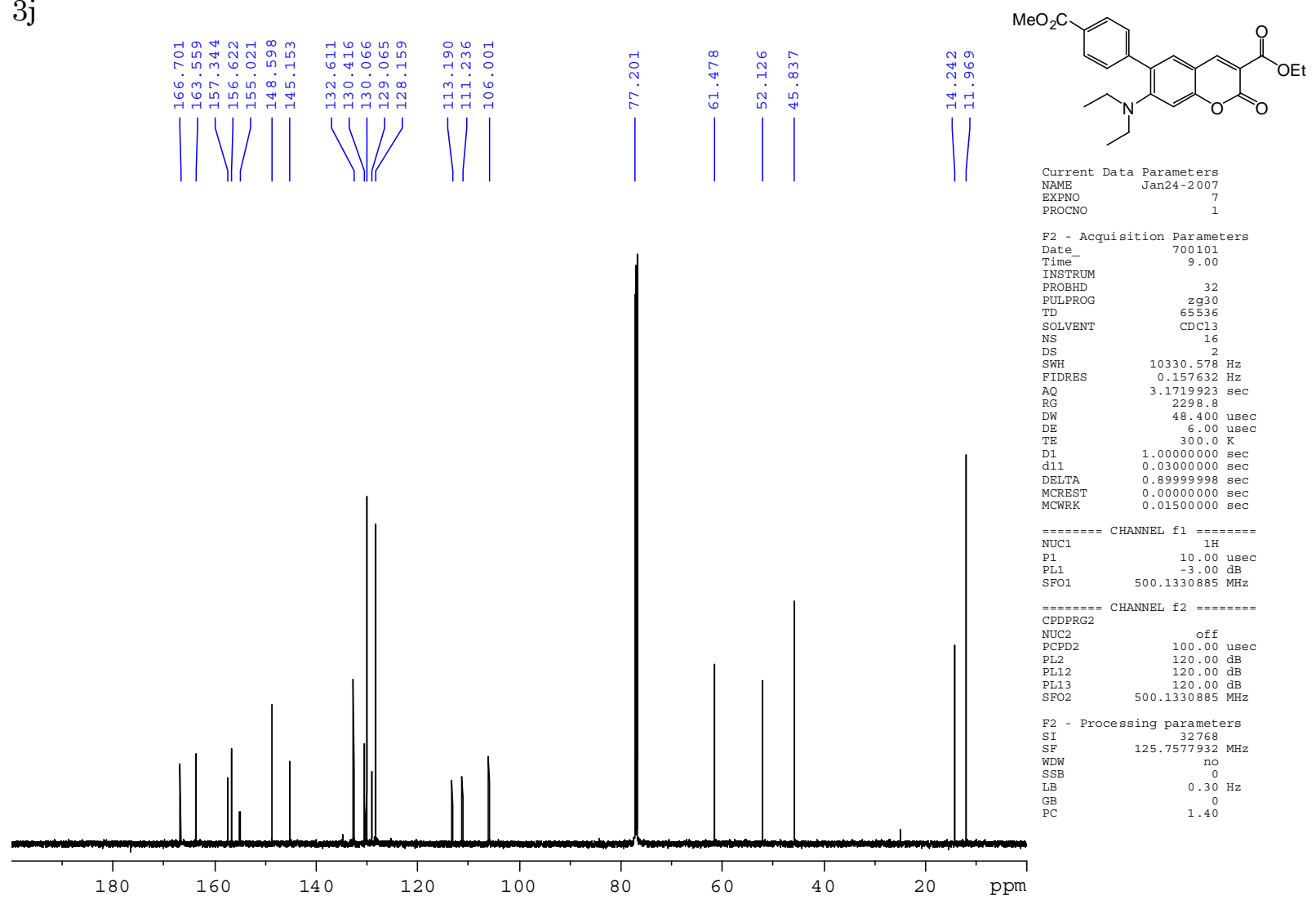
$3 \mathrm{k}$
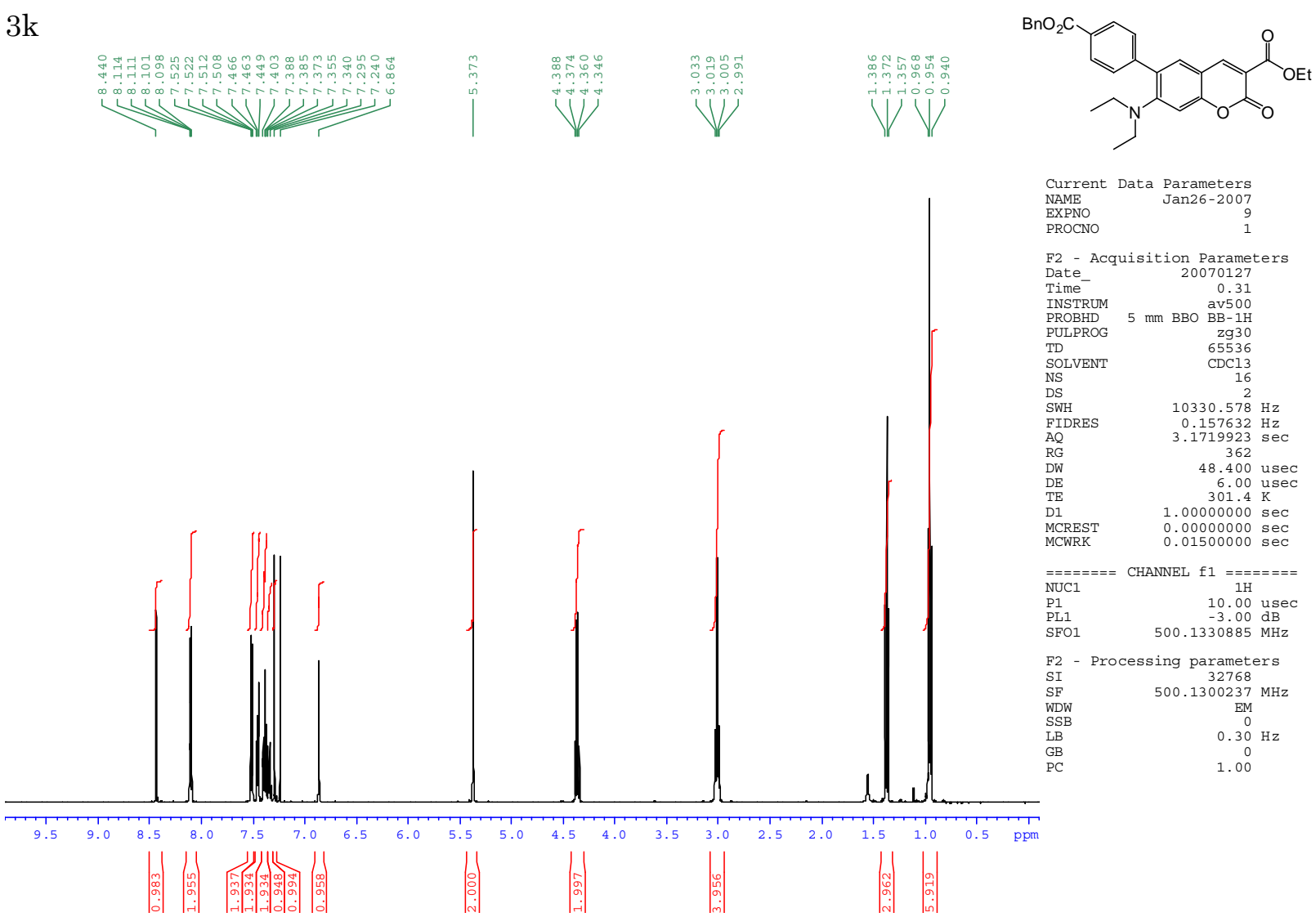

$3 \mathrm{k}$
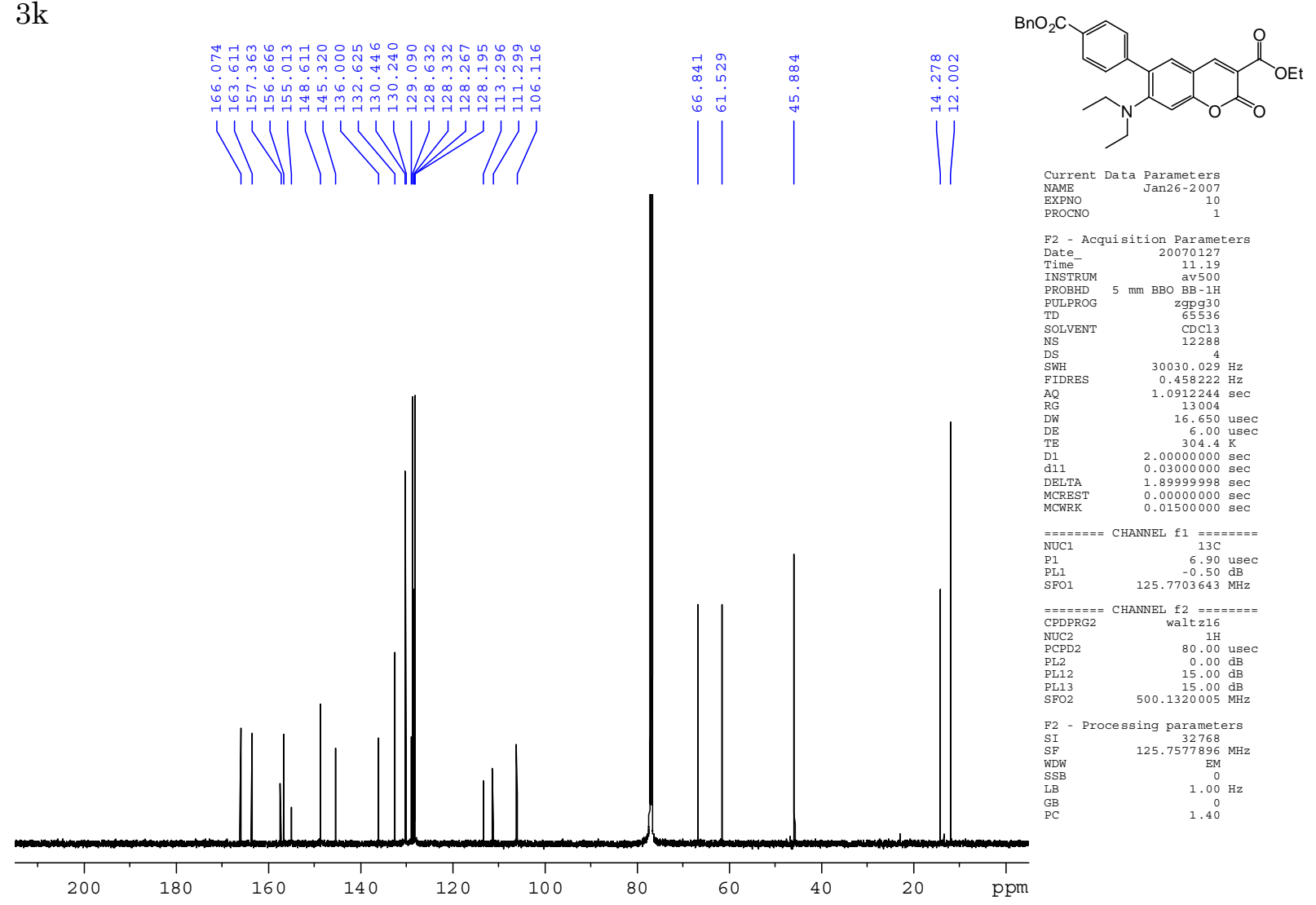
31
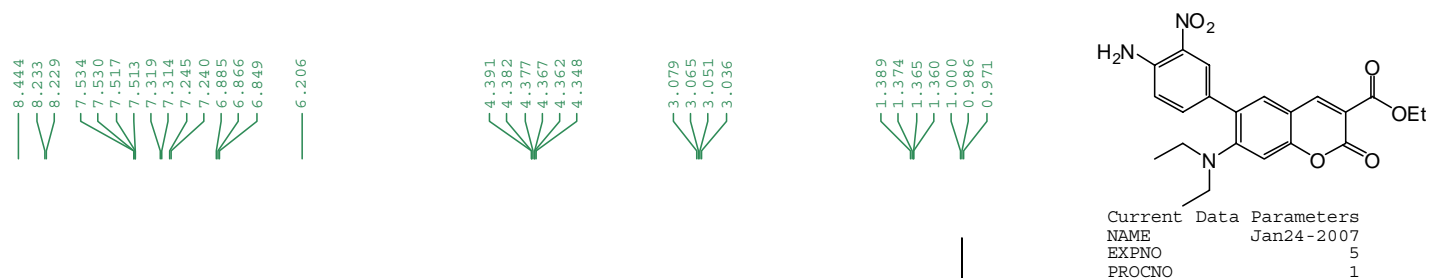

F2 - Acquisition Parameters

Date_r 20070124

Time
INSTRUM

PROBHD $5 \mathrm{~mm}$ BBO $\mathrm{BB}-1 \mathrm{H}$

PULPROG
zg30

TD

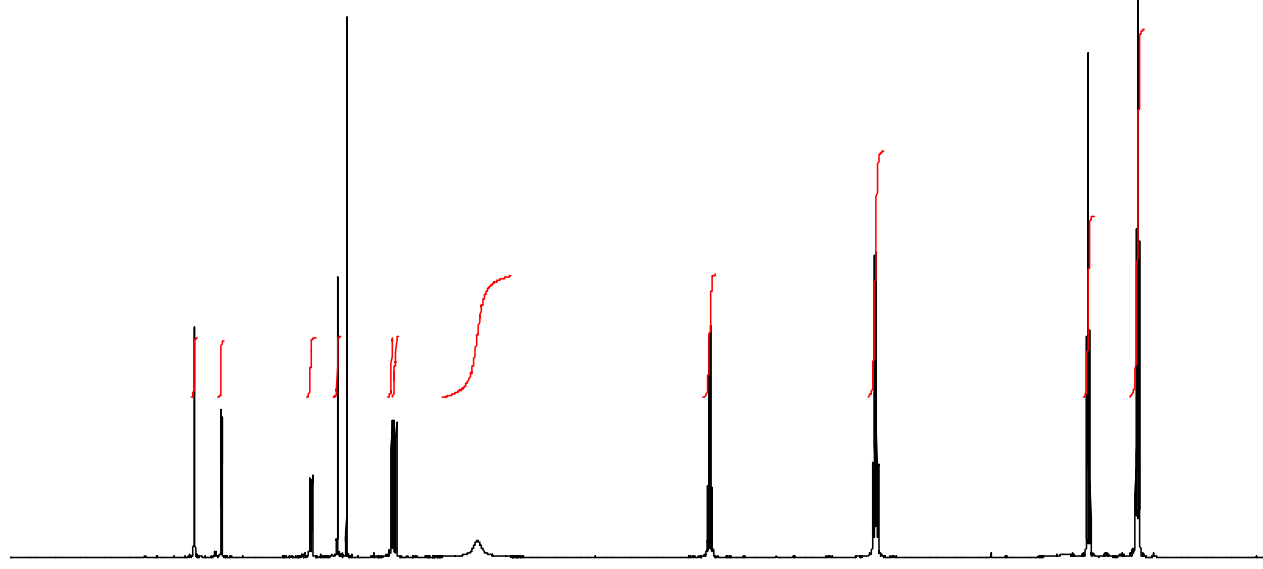

NS

DS

$\mathrm{FQ}$
$\mathrm{RG}$

$\mathrm{DW}$
$\mathrm{DE}$
$\mathrm{TE}$

D1

MCWRK

$0.01500000 \mathrm{sec}$

$=======$ CHANNEL $\mathrm{f} 1=======$
NUC1

$\begin{array}{ll}\text { P1 } & 1 \mathrm{H} \text { usec } \\ \text { PL1 } & 10.00 \mathrm{usec}\end{array}$

SFO1 $\quad 500.1330885 \mathrm{MHz}$

$\begin{array}{lr}\text { F2 } & \text { - Processing parameters } \\ \text { SI } & 32768 \\ \mathrm{SF} & 500.1300237 \mathrm{MHz}\end{array}$

SF $\quad 500.1300237 \mathrm{MH}$

$\begin{array}{lc}\text { WDW } & \text { EM } \\ \text { SSB } & 0 \\ \text { LB } & 0.30 \mathrm{~Hz} \\ \text { GB } & 0 \\ \text { PC } & 1.00\end{array}$

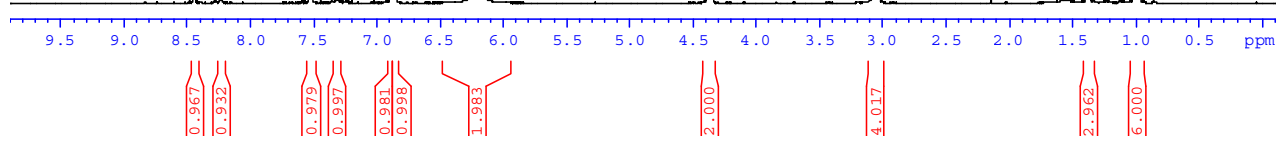

31

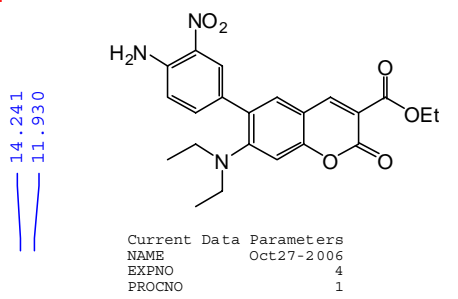

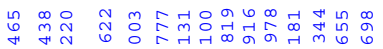

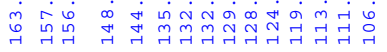

| | | | |V//I ||

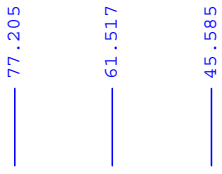

F2
Da
Ti
IN
I
P
P
TD
S
S
NS
D
S
F
A
A
R
D
D
T
D
d
D
D

F2 - Acquisition Parameter

20061028
Time

INSTRUM
PROBHD
PULPROG $\quad \begin{array}{r}\text { av } 500 \\ \text { BBO } \mathrm{BB}-1 \mathrm{H} \\ \text { zgpg30 }\end{array}$

$\begin{array}{lr}\text { Pgpg30 } \\ \text { PDDROG } & 65536 \\ \text { SOLVENT } & \mathrm{CDCl} \\ & 1024\end{array}$

$\begin{array}{lc}\text { SOLVENT } & \text { CDCl3 } \\ \text { NS } & 1024 \\ \text { DS } & 8 \\ \text { SWH } & 30030.029 \mathrm{~Hz} \\ \text { FTDRES } & 0.458222 \mathrm{~Hz}\end{array}$

$\begin{array}{ll}\text { ADRES } & 0.458222 \mathrm{~Hz} \\ \mathrm{AC} & 1.0912244 \mathrm{sec} \\ & 10321.3 \mathrm{sec}\end{array}$

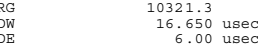

$\begin{array}{ll} & 301.8 \mathrm{~K} \\ \mathrm{DE} & 2.00000000 \mathrm{sec}\end{array}$

$\begin{array}{ll}\text { DELTA } & 1.89999998 \mathrm{sec} \\ \text { MCREST } & 0.00000000 \mathrm{sec}\end{array}$

fHANNEL f $1====== \pm$

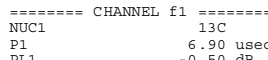

$\begin{array}{lr}\text { P1 } & 6.90 \mathrm{usec} \\ \text { PL1 } & -0.50 \mathrm{~dB} \\ \text { SFO1 } & 125.7703643 \mathrm{MHz}\end{array}$

$\begin{array}{lc}====== & \text { CHANNEL } \mathrm{f} 2======= \\ \text { CPDPRG2 } & \text { walt z16 } \\ \text { NUC2 } & 1 \mathrm{H} \\ \text { PCPD2 } & 80.00 \mathrm{usec} \\ \text { PL2 } & 0.00 \mathrm{~dB} \\ \text { PL12 } & 15.00 \mathrm{~dB}\end{array}$

$\begin{array}{lr}\text { PL12 } & 15.00 \mathrm{~dB} \\ \text { PL13 } & 15.00 \mathrm{~dB} \\ \text { SFO2 } & 500.1320005 \mathrm{MHz}\end{array}$

F2 - Processing parameters
SI
SF

$\begin{array}{lc}\text { SI } & 32768 \\ \text { SE } & 125.7577951 \mathrm{MHz} \\ \text { WDW } & \mathrm{EM} \\ \text { SSB } & 0 \\ \text { LB } & 1.00 \mathrm{~Hz} \\ \text { GB } & 0 \\ \text { PC } & 1.40\end{array}$

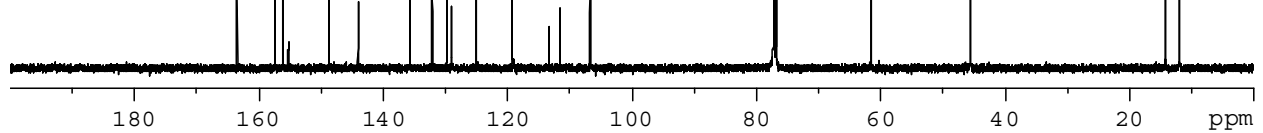


$3 \mathrm{~m}$
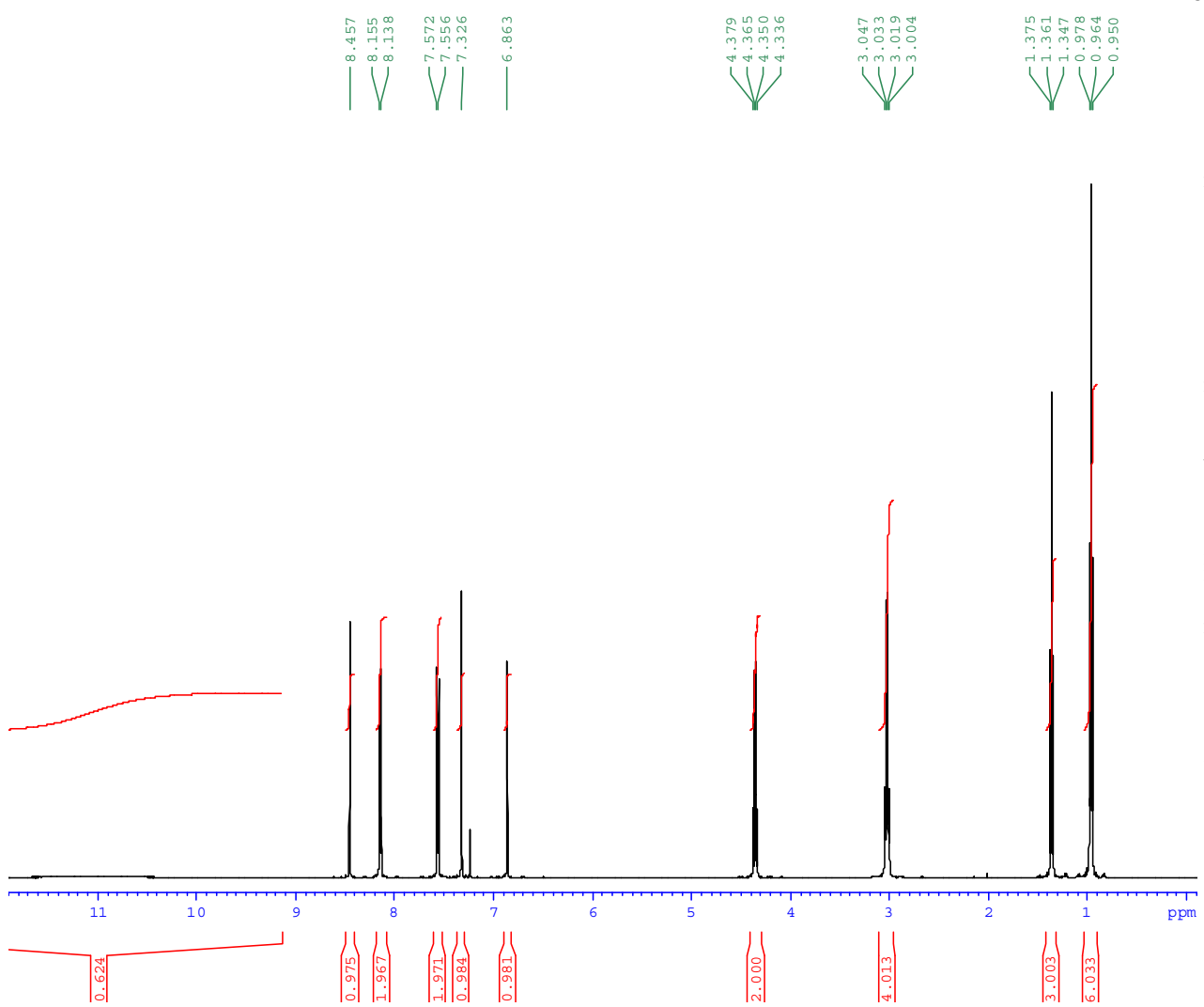

$3 \mathrm{~m}$

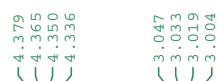

mं लंm

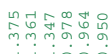

Vij
$\mathrm{HO}_{2}$

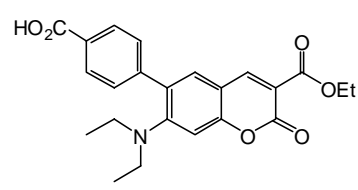

$\begin{array}{lr}\text { Current Data } & \text { Parameters } \\ \text { NAME } & \text { Jan27-2007 } \\ \text { EXPNO } & 3\end{array}$

ROCNO 1

F2 - Acquisition Parameters

Date__ 20070127

$\begin{array}{ll}\text { Time } & 16.48 \\ \text { INSTRUM } & \text { av500 }\end{array}$

PROBHD $5 \mathrm{~mm}$ BBO BB-1H

PULPROG $\quad \mathrm{zg}^{30}$

TD

SOLVEN
NS

$\begin{array}{lr}\text { DS } & 2 \\ \text { SWH } & 10330.578 \mathrm{~Hz} \\ \text { FIDRES } & 0.157632 \mathrm{~Hz}\end{array}$

$\begin{array}{ll}\text { AQ } & 0.157632 \mathrm{~Hz} \\ \text { RG } & 3.1719923 \mathrm{sec}\end{array}$

$\begin{array}{lr}\mathrm{RW} & 71.8 \mathrm{u} \\ \mathrm{DW} & 48.400 \mathrm{usec}\end{array}$

TE

$1.00000000 \mathrm{sec}$

$\begin{array}{ll}\text { MCREST } & 0.00000000 \mathrm{sec} \\ \text { MCWRK } & 0.01500000 \mathrm{sec}\end{array}$

$=======$ CHANNEL $\mathrm{f} 1 \mathrm{~s}=======$

NUT1

P1 1

SFO1

F2
$\mathrm{SI}$
$\mathrm{SF}$

SF
WDW
SSB

SSB
LB
GB
PC
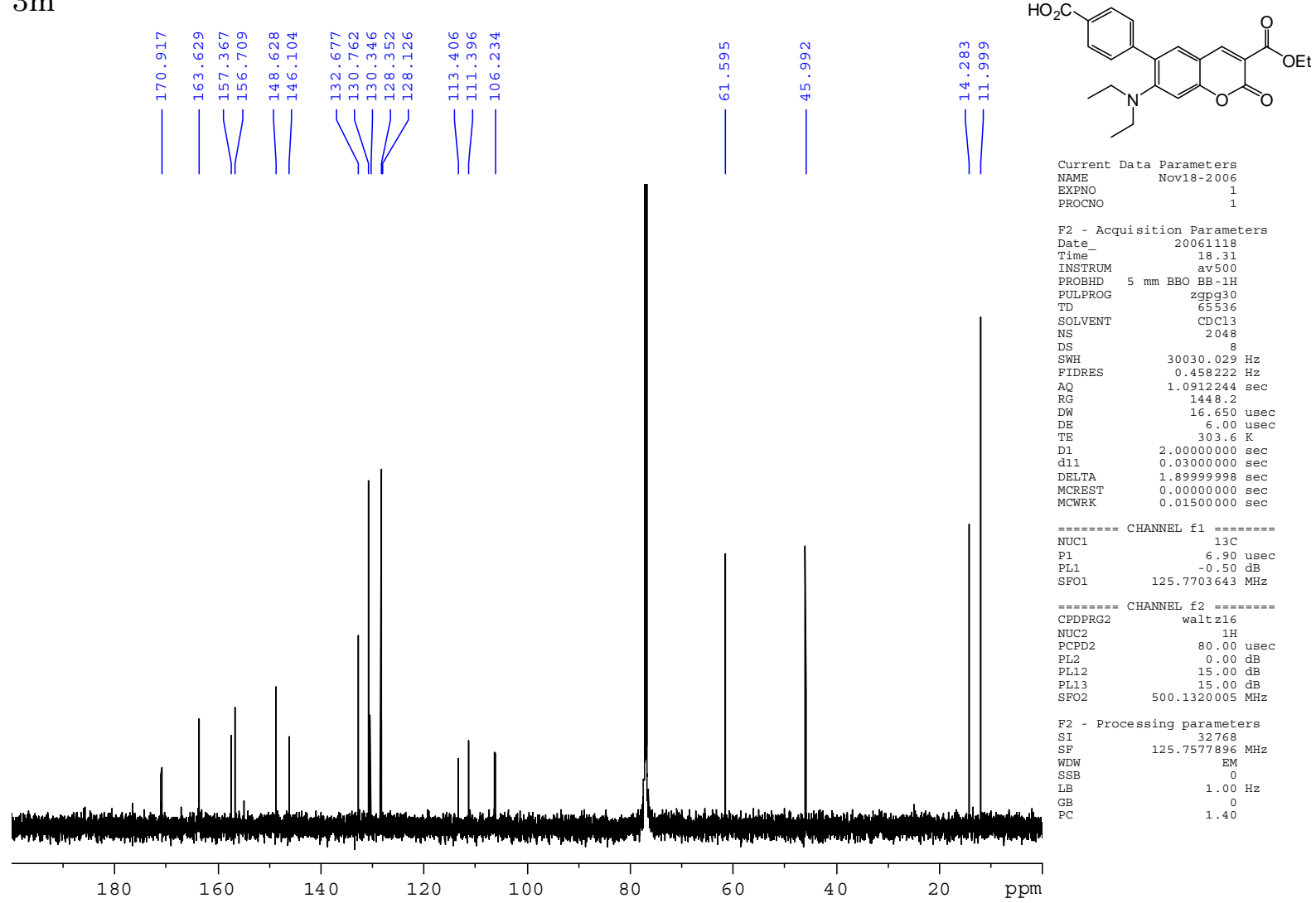

F2 - Acquisition Parameter

20061118

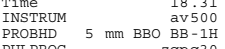

$\begin{array}{lr}\text { PULPROG } & \text { zgpg30 } \\ \text { TD } & 65536 \\ \text { SOLVENT } & \text { CDC13 } \\ & 2048\end{array}$

$\begin{array}{lc}\text { NS } & \text { CDCl3 } \\ \text { NS } & 2048 \\ \text { DSH } & 30030.029 \mathrm{~Hz} \\ \text { SWI } & 0.458222 \mathrm{~Hz}\end{array}$

$\begin{array}{ll}\text { NWH } & 30030.029 \mathrm{~Hz} \\ \text { FIDRES } & 0.458222 \mathrm{~Hz} \\ \text { NQ } & 1.0912244 \mathrm{sec} \\ & 1448.2\end{array}$

144.2
RG
DW
$6.650 \mathrm{usec}$
$6.00 \mathrm{usec}$

$303.6 \mathrm{~K}$
$\mathrm{D}$

$\begin{array}{ll}2.000990 \mathrm{sec} \\ \text { DELTA } & 1.89999998 \mathrm{sec}\end{array}$

$\begin{array}{ll}\text { MCREST } & 0.00000000 \mathrm{sec} \\ \text { MCWRK } & 0.01500000 \mathrm{sec}\end{array}$

$======$ CHANNEL f1 $\begin{aligned} & ======= \\ & 13 \mathrm{C}\end{aligned}$
NUC1

$\begin{array}{lr}\text { P1 } & 6.90 \mathrm{usec} \\ \text { PL1 } & -0.50 \mathrm{~dB} \\ \text { SFO1 } & 125.7703643 \mathrm{MHz}\end{array}$

$=======$ CHANNEL $\mathrm{f} 2=======$
walt $\mathrm{z} 16$
CPDPRG2
$1 \mathrm{H}$

$\begin{array}{ll}\text { NUC2 } & 1 \mathrm{H} \\ \text { PCPD2 } & 80.00 \mathrm{usec} \\ \text { PL2 } & 0.00 \mathrm{~dB} \\ \text { PL12 } & 15.00 \mathrm{~dB}\end{array}$

$\begin{array}{lr}\text { PL12 } & 15.00 \mathrm{~dB} \\ \text { PL13 } & 15.00 \mathrm{~dB} \\ \text { S102 } & 500.1320005 \mathrm{MH}\end{array}$

$\begin{array}{rr}\text { F2 } & \text { Processing parameters } \\ \mathrm{SI} & 32768 \\ \mathrm{SF} & 125.7577896 \mathrm{MHz}\end{array}$

SF
WD
SSB
LB
GB

$\mathrm{LB}$
$\mathrm{GB}$
$\mathrm{PC}$

$125.7577896 \mathrm{MHz}$
$\mathrm{EM}$

$7577896 \mathrm{MH}$
$\mathrm{EM}$
0
$1.00 \mathrm{~Hz}$
0 
$3 n$
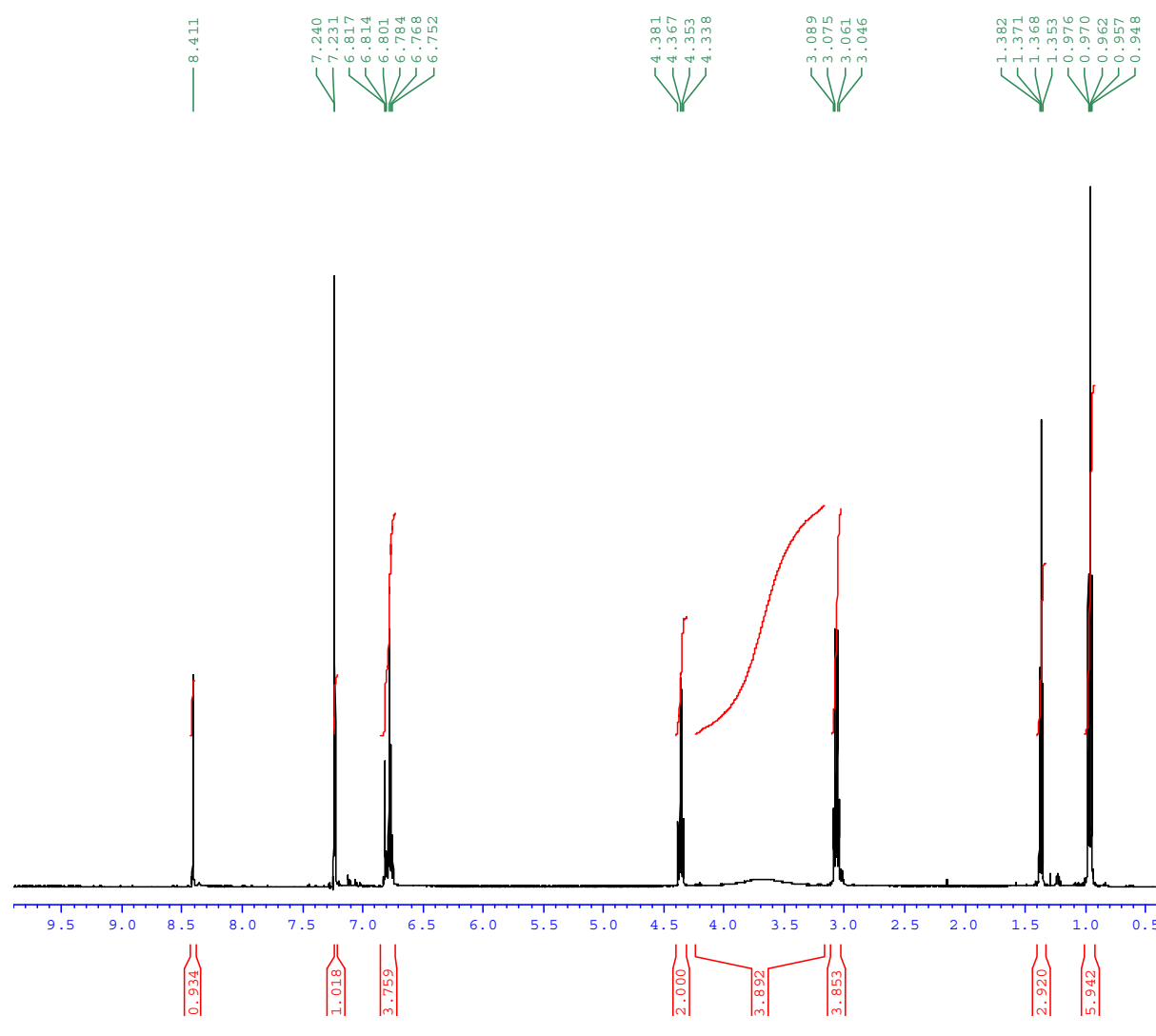

$3 n$
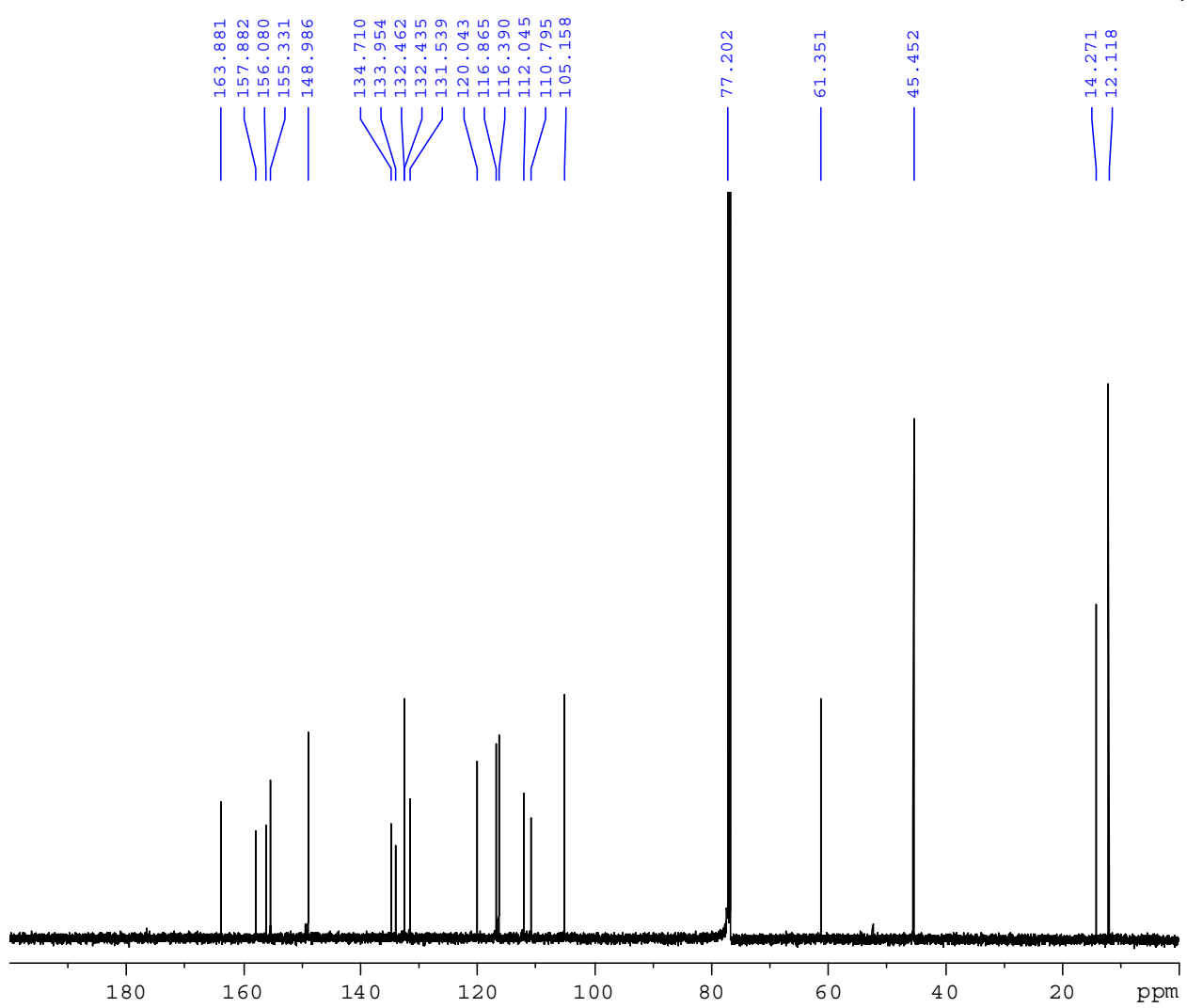

$\mathrm{S} 25$

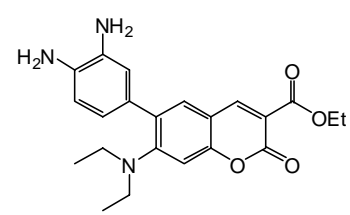

$\begin{array}{ll}\text { Current Data } & \text { Parameters } \\ \text { NAME } & \text { Jan25-2007 }\end{array}$

PROCNO

F2 - Acquisition Parameters

Date_ 20070125

Time $\quad 23.05$

PROBHD $5 \mathrm{~mm}$ BBO BB-1H

\begin{tabular}{lr} 
PULPROG & $\mathrm{zg} 30$ \\
\hline & 65536
\end{tabular}

TD

SOLVEN

NS
DS
SWH

$\begin{array}{lr}2 \\ \text { SWH } & 10330.578 \mathrm{~Hz}\end{array}$

$\begin{array}{ll}\text { FIDRES } & 0.157632 \mathrm{~Hz} \\ \mathrm{AQ} & 3.1719923 \mathrm{sec}\end{array}$

AQ
RW
DW

$\mathrm{DW}$
$\mathrm{DE}$
$\mathrm{TE}$

DI

MCWRK

456.1
48.400 use

48.400 usec
6.00 usec

$.00000000 \mathrm{sec}$
$.00000000 \mathrm{sec}$

$0.01500000 \mathrm{sec}$

$=======$ CHANNEL $\mathrm{f} 1========$
NUC1

$\begin{array}{ll}\text { NUC1 } & 1 \mathrm{H} \\ \text { P1 } & 10.00 \mathrm{usec} \\ \text { PL1 } & -3.00 \mathrm{~dB}\end{array}$

$-3.00 \mathrm{~dB}$
$\mathrm{PLO1}$
$500.1330885 \mathrm{MHz}$

$\begin{array}{lr}\text { F2 } & \text { - Processing parameters } \\ \text { SI } & 32768 \\ \text { SF } & 500.1300237 \mathrm{MHz}\end{array}$

$\begin{array}{lc}\text { SF } & 500.1300237 \\ \text { WDW } & \text { EM } \\ \text { SSB } & 0\end{array}$

$\begin{array}{lc}\text { SSB } & 0 \\ \mathrm{LB} & 0.30 \mathrm{~Hz} \\ \mathrm{~GB} & 0\end{array}$

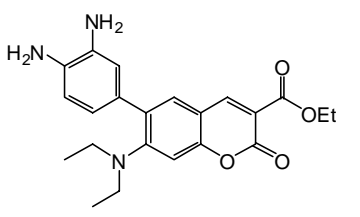

$\begin{array}{lr}\text { Current } & \text { Data } \\ \text { NAME } & \text { Nameters } \\ \text { EXPNO } & \text { Nov3-2006 } \\ \text { PROCNO } & 4 \\ & 1\end{array}$

F2 - Acquisition Parameters

$\begin{array}{lr}\text { Date_ } & \text { Acquisition Parame } \\ \text { Time } & 20061104 \\ \text { The } & 1.28\end{array}$

av500
NSTRUM $5 \mathrm{~mm} \mathrm{BBO} \mathrm{BB-1H}$

$2 \mathrm{~mm}$ BBO $\mathrm{BB}-1 \mathrm{H}$
$2 \mathrm{gpg} 30$

CDC13
OLLENT
1024

$\begin{array}{lr}\text { DS } & 102 \\ \text { SWH } & 30030.029 \mathrm{~Hz} \\ \text { IDRES } & 0.458222 \mathrm{~Hz}\end{array}$

$1.0912244 \mathrm{sec}$
4597.6

45.650 usec
6.00 usec
30.08

$\begin{array}{ll}2.00001 .8 \mathrm{~K} \\ 1 & 0.000000 \mathrm{sec}\end{array}$

$\begin{array}{ll}\text { DELTA } & 1.89999998 \mathrm{sec} \\ \text { MCREST } & 0.0000000 \mathrm{sec}\end{array}$

$1.89000000 \mathrm{sec}$
$0.01500000 \mathrm{sec}$

$=======$ CHANNEL f1 $=======$
NUC1
$13 \mathrm{C}$

$\begin{array}{lr}\text { P1 } & 6.90 \text { usec } \\ \text { PL1 } & -0.50 \mathrm{~dB}\end{array}$

$======\quad$ CHANNEL f $2 \quad=======$
walt 216

$\begin{array}{lc}\text { NUC2 } & 1 \mathrm{H} \\ \text { PCDD2 } & 80.00 \mathrm{usec} \\ \text { PL2 } & 0.00 \mathrm{~dB} \\ & 15.00 \mathrm{~dB}\end{array}$

$15.00 \mathrm{~dB}$
$\mathrm{~L} 12$
$\mathrm{L13}$

2 - Processing parameters

$\begin{array}{lc}\text { SI } & 32768 \\ \text { SF } & 125.7577951 \mathrm{MHz} \\ \text { WDW } & \text { EM }\end{array}$

$\begin{array}{lr}\text { WDW } & 125.7577951 \mathrm{MHz} \\ \text { SSB } & 0\end{array}$ 
30

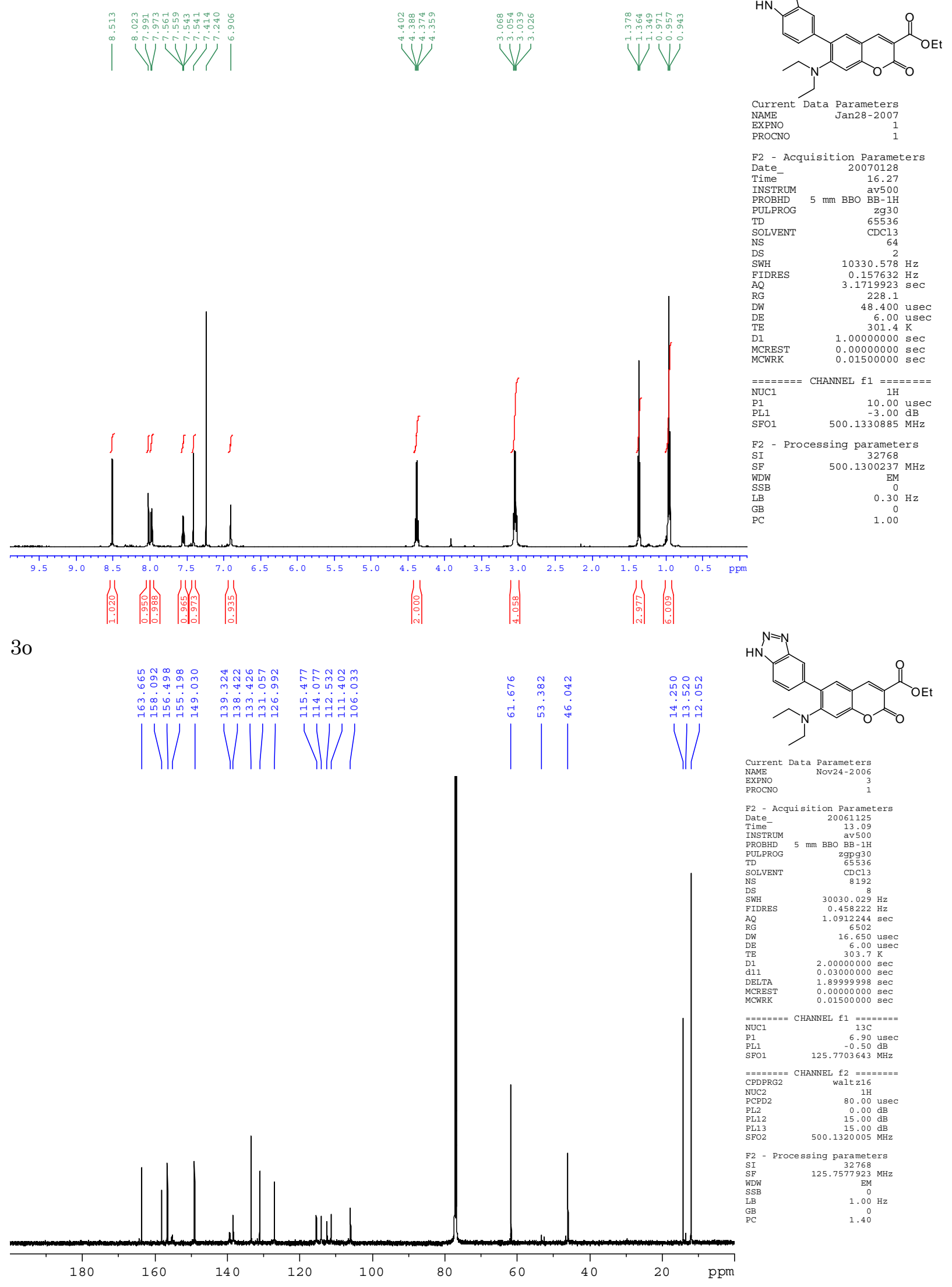

